OPEN ACCESS

University of Dundee

\title{
A multi-layer functional genomic analysis to understand noncoding genetic variation in lipids
}

Smith, Blair H.; Palmer, Colin; Zhu, Xiang; Brown, Christopher D

DOI:

10.1101/2021.12.07.470215

Publication date:

2021

Document Version

Early version, also known as pre-print

Link to publication in Discovery Research Portal

Citation for published version (APA):

Smith, B. H., Palmer, C., Zhu, X., \& Brown, C. D. (2021). A multi-layer functional genomic analysis to understand noncoding genetic variation in lipids. BioRxiv. https://doi.org/10.1101/2021.12.07.470215

\section{General rights}

Copyright and moral rights for the publications made accessible in Discovery Research Portal are retained by the authors and/or other copyright owners and it is a condition of accessing publications that users recognise and abide by the legal requirements associated with these rights.

- Users may download and print one copy of any publication from Discovery Research Portal for the purpose of private study or research.

- You may not further distribute the material or use it for any profit-making activity or commercial gain.

- You may freely distribute the URL identifying the publication in the public portal.

Take down policy

If you believe that this document breaches copyright please contact us providing details, and we will remove access to the work immediately and investigate your claim. 
1 A multi-layer functional genomic analysis to understand noncoding genetic variation in

\section{2 lipids}

3 Shweta Ramdas ${ }^{1 *}$, Jonathan Judd ${ }^{2 *}$, Sarah E Graham ${ }^{3 *}$, Stavroula Kanoni ${ }^{* *}$, Yuxuan Wang ${ }^{5^{*}}$, Ida

4 Surakka ${ }^{3}$, Brandon Wenz', Shoa L Clarke ${ }^{6,7}$, Alessandra Chesi' ${ }^{8}$, Andrew Wells ${ }^{1}$, Konain Fatima

5 Bhatti ${ }^{4}$, Sailaja Vedantam ${ }^{910}$, Thomas W Winkler ${ }^{11}$, Adam E Locke ${ }^{12}$, Eirini Marouli ${ }^{4}$, Greg JM

6 Zajac $^{13}$, Kuan-Han $\mathrm{H} \mathrm{Wu}^{14}$, Ioanna Ntalla ${ }^{15}$, Qin Hui ${ }^{16,17}$, Derek Klarin ${ }^{18,1920}$, Austin T Hilliard ${ }^{6}$,

7 Zeyuan Wang ${ }^{16,17}$, Chao Xue ${ }^{3}$, Gudmar Thorleifsson" ${ }^{21}$, Anna Helgadottir ${ }^{21}$, Daniel F

8 Gudbjartsson $^{21.22}$, Hilma Holm ${ }^{21}$, Isleifur Olafsson ${ }^{23}$, Mi Yeong Hwang ${ }^{24}$, Sohee Han $^{24}$, Masato

$9 \quad$ Akiyama $^{25,26}$, Saori Sakaue ${ }^{2728,29}$, Chikashi Terao ${ }^{30}$, Masahiro Kanai ${ }^{25,3132}$, Wei Zhou ${ }^{33,1420,}$, Ben M

10 Brumpton ${ }^{34353.36}$, Humaira Rasheed ${ }^{34,3537}$, Aki S Havulinna ${ }^{38.39}$, Yogasudha Veturi ${ }^{40}$, Jennifer Allen

11 Pacheco ${ }^{41}$, Elisabeth A Rosenthal ${ }^{42}$, Todd Lingren ${ }^{43}$, QiPing Feng ${ }^{44}$, Iftikhar J. Kullo ${ }^{45}$, Akira

$12 \mathrm{Narita}^{46}$, Jun Takayama ${ }^{46}$, Hilary C $\mathrm{Martin}^{47}$, Karen A Hunt ${ }^{48}$, Bhavi Trivedii ${ }^{48}$, Jeffrey Haessler ${ }^{49}$,

13 Franco Giulianini ${ }^{50}$, Yuki Bradford ${ }^{40}$, Jason E Miller ${ }^{40}$, Archie Campbell ${ }^{51,52}$, Kuang Lin ${ }^{53}$, Iona Y

14 Millwood $^{53,5}$, Asif Rasheed ${ }^{5 s}$, George Hindy $y^{56}$, Jessica D Faul ${ }^{57}$, Wei Zhao ${ }^{58}$, David R Weiir ${ }^{57}$,

15 Constance Turman ${ }^{59}$, Hongyan Huang ${ }^{59}$, Mariaelisa Graff ${ }^{60}$, Ananyo Choudhury ${ }^{61}$, Dhriti

16 Sengupta $^{61}$, Anubha Mahajan $^{62}$, Michael R Brown ${ }^{63}$, Weihua Zhang ${ }^{6,45,66,}$, Ketian Yu ${ }^{13}$, Ellen M

17 Schmidt $^{13}$, Anita Pandit ${ }^{13}$, Stefan Gustafsson ${ }^{67}$, Xianyong Yin ${ }^{13}$, Jian'an Luan ${ }^{68}$, Jing-Hua Zhao ${ }^{69}$,

18 Fumihiko Matsuda ${ }^{70}$, Hye-Mi Jang ${ }^{24}$, Kyungheon Yoon $^{24}$, Carolina Medina-Gomez ${ }^{71}$, Achilleas

19 Pitsillides $^{5}$, Jouke Jan Hottenga ${ }^{72,73}$, Andrew R Wood ${ }^{74}$, Yingji Ji ${ }^{74}$, Zishan Gao ${ }^{75,76,77}$, Simon

20 Haworth $^{35,78}$, Ruth E Mitchell ${ }^{35,79}$, Jin Fang Chai ${ }^{80}$, Mette Aadahl ${ }^{81.12}$, Anne A Bjerregaard ${ }^{81}$, Jie

$21 \mathrm{YaO}^{83}$, Ani Manichaikul ${ }^{84}, \mathrm{PhD}$ Wen-Jane ${ }^{85}, \mathrm{PhD}$ Chao A ${ }^{86}$, Helen R Warren ${ }^{87.88}$, Julia Ramirez ${ }^{87}$,

22 Jette Bork-Jensen ${ }^{89}$, Line L Kårhus ${ }^{81}$, Anuj Goel ${ }^{00,9}$, Maria Sabater-Lleal ${ }^{2093}$, Raymond

23 Noordam $^{94}$, Pala Mauro ${ }^{95}$, Floris Matteo ${ }^{96.95}$, Aaron F McDaid ${ }^{9798}$, Pedro Marques-Vidal ${ }^{99}$,

24 Matthias Wielscher ${ }^{100}$, Stella Trompet ${ }^{101.102}$, Naveed Sattar ${ }^{103}$, Line T Møllehave ${ }^{81}$, Matthias

25 Munz $^{104}$, Lingyao Zeng ${ }^{105,106}$, Jianfeng Huang ${ }^{107}$, Bin Yang ${ }^{107}$, Alaitz Poveda ${ }^{108}$, Azra Kurbasic ${ }^{108}$, 
26 Sebastian Schönherr ${ }^{109}$, Lukas Forer ${ }^{109}$, Markus Scholz ${ }^{10,111}$, Tessel E. Galesloot ${ }^{112}$, Jonathan P.

27 Bradfield ${ }^{113}$, Sanni E Ruotsalainen ${ }^{38}$, E Warwick Daw ${ }^{114}$, Joseph M Zmuda ${ }^{115}$, Jonathan S

28 Mitchell ${ }^{116}$, Christian Fuchsberger ${ }^{116}$, Henry Christensen" ${ }^{117}$, Jennifer A Brody ${ }^{118}$, Phuong Le ${ }^{119,120}$,

29 Mary F Feitosa ${ }^{114}$, Mary K Wojczynski ${ }^{14}$, Daiane Hemerich ${ }^{121}$, Michael Preuss ${ }^{121}$, Massimo

30 Mangino $^{12,123}$, Paraskevi Christofidou ${ }^{122}$, Niek Verweij ${ }^{124}$, Jan W Benjamins ${ }^{124}$, Jorgen

31 Engmann ${ }^{15,126}$, Tsao L. Noah ${ }^{127}$, Anurag Verma ${ }^{1}$, Roderick C Slieker ${ }^{128,129}$, Ken Sin Lo ${ }^{130}$, Nuno R

$32 Z_{\text {Zilhao }}^{131}$, Marcus E Kleber ${ }^{132,133}$, Graciela E Delgado ${ }^{132}$, Shaofeng Huo ${ }^{134}$, Daisuke D Ikeda ${ }^{135}$,

33 Hiroyuki Iha ${ }^{135}$, Jian Yang ${ }^{136,137,138}$, Jun Liu ${ }^{139,140}$, Ayșe Demirkan ${ }^{141,142}$, Hampton L Leonard ${ }^{143,14}$,

34 Jonathan Marten ${ }^{145}$, Carina Emmel ${ }^{146}$, Börge Schmidt ${ }^{146}$, Laura J Smyth ${ }^{147}$, Marisa Cañadas-

35 Garre ${ }^{147,148,149,150}$, Chaolong Wang ${ }^{151.152}$, Masahiro Nakatochi ${ }^{153}$, Andrew Wong ${ }^{154}$, Nina Hutri-

36 Kähönen ${ }^{155}$, Xueling Sim ${ }^{80}$, Rui Xia ${ }^{156}$, Alicia Huerta-Chagoya ${ }^{157}$, Juan Carlos Fernandez-

37 Lopez $^{158}$, Valeriya Lyssenko ${ }^{159,160}$, Suraj S Nongmaithem ${ }^{161}$, Alagu Sankareswaran ${ }^{161,162}$,

38 Marguerite R Irvin ${ }^{154}$, Christopher Oldmeadow ${ }^{163}$, Han-Na Kim ${ }^{164,165}$, Seungho Ryu ${ }^{16,67,}$, Paul RHJ

39 Timmers $^{168.145}$, Liubov Arbeeva ${ }^{169}$, Rajkumar Dorajoo ${ }^{152.170}$, Leslie A Lange ${ }^{171}$, Gauri Prasad ${ }^{172,173}$,

40 Laura Lorés-Motta ${ }^{174}$, Marc Pauper ${ }^{174}$, Jirong Long ${ }^{175}$, Xiaohui Li ${ }^{83}$, Elizabeth Theusch ${ }^{176}$,

41 Fumihiko Takeuchi ${ }^{177}$, Cassandra N Spracklen ${ }^{17,179}$, Anu Loukola ${ }^{38}$, Sailalitha Bollepalli ${ }^{38}$,

42 Sophie C Warner ${ }^{180.181}$, Ya Xing Wang ${ }^{182}$, Wen B. Wei ${ }^{183}$, Teresa Nutile ${ }^{184}$, Daniela Ruggiero ${ }^{184.185}$,

43 Yun Ju Sung ${ }^{186}$, Shufeng Chen ${ }^{107}$, Fangchao Liu ${ }^{107}$, Jingyun Yang ${ }^{187,188}$, Katherine A Kentistou ${ }^{168}$,

44 Bernhard Banas ${ }^{199}$, Anna Morgan ${ }^{190}$, Karina Meidtner ${ }^{191,192}$, Lawrence F Bielak ${ }^{58}$, Jennifer A

45 Smith $^{58.57}$, Prashantha Hebbar ${ }^{193}$, Aliki-Eleni Farmaki ${ }^{194.195}$, Edith Hofer ${ }^{196,197}$, Maoxuan Lin ${ }^{198}$, Maria

46 Pina Concas ${ }^{199}$, Simona Vaccargiu ${ }^{200}$, Peter J van der Most $^{201}$, Niina Pitkänen ${ }^{202203}$, Brian E

47 Cade $^{204.205}$, Sander W. van der Laan ${ }^{206}$, Kumaraswamy Naidu Chitrala ${ }^{207208}$, Stefan Weiss ${ }^{209}$, Amy

48 R Bentley ${ }^{210}$, Ayo P Doumatey ${ }^{210}$, Adebowale A Adeyemo ${ }^{210}$, Jong Young Lee ${ }^{211}$, Eva RB

49 Petersen $^{212}$, Aneta A Nielsen ${ }^{213}$, Hyeok Sun Choi $^{214}$, Maria Nethander ${ }^{2152.26}$, Sandra Freitag-Wolf ${ }^{217}$,

50 Lorraine Southam ${ }^{21847}$, Nigel W Rayner ${ }^{219220,47218}$, Carol A Wang ${ }^{211}$, Shih-Yi Lin ${ }^{222223,224}$, Jun-Sing 
51 Wang ${ }^{225,226}$, Christian Couture ${ }^{227}$, Leo-Pekka Lyytikäinen ${ }^{28,229}$, Kjell Nikus ${ }^{230.231}$, Gabriel Cuellar-

52 Partida $^{232}$, Henrik Vestergaard ${ }^{89233}$, Bertha Hidalgo ${ }^{234}$, Olga Giannakopoulou ${ }^{4}$, Qiuyin Cai ${ }^{175}$,

53 Morgan O Obura ${ }^{128}$, Jessica van Setten ${ }^{235}$, Karen Y. He ${ }^{236}$, Hua Tang ${ }^{237}$, Natalie Terzikhan ${ }^{238}$, Jae

54 Hun Shin ${ }^{214}$, Rebecca D Jackson ${ }^{239}$, Alexander P Reiner ${ }^{240}$, Lisa Warsinger Martin ${ }^{241}$, Zhengming

55 Chen ${ }^{53,44}$, Liming $\mathrm{Li}^{242}$, Takahisa Kawaguchi ${ }^{70}$, Joachim Thiery ${ }^{243,111}$, Joshua C Bis ${ }^{118}$, Lenore J

56 Launer $^{244}$, Huaixing Li ${ }^{245}$, Mike A Nalls ${ }^{143.144}$, Olli T Raitakari ${ }^{246.247 .248}$, Sahoko Ichihara ${ }^{249}$, Sarah H

57 Wild $^{250}$, Christopher P Nelson ${ }^{180.181}$, Harry Campbell ${ }^{168}$, Susanne Jäger ${ }^{191,192}$, Toru Nabika ${ }^{251}$, Fahd

58 Al-Mulla ${ }^{193}$, Harri Niinikoskii ${ }^{252.253}$, Peter S Braund ${ }^{180,181}$, Ivana Kolcicicis4, Peter Kovacs ${ }^{255}$, Tota

59 Giardoglou ${ }^{256}$, Tomohiro Katsuya ${ }^{257.258}$, Dominique de Kleijn ${ }^{259}$, Gert J. de Borst ${ }^{259}$, Eung Kweon

$60 \mathrm{Kim}^{260}$, Hieab H.H. Adams ${ }^{238261}$, M. Arfan Ikram ${ }^{238}$, Xiaofeng Zhu ${ }^{236}$, Folkert W Asselbergs ${ }^{235}$,

61 Adriaan O Kraaijeveld ${ }^{235}$, Joline WJ Beulens ${ }^{128.262}$, Xiao-Ou Shu ${ }^{175}$, Loukianos S Rallidis ${ }^{263}$, Oluf

62 Pedersen $^{89}$, Torben Hansen ${ }^{89}$, Paul Mitchell ${ }^{264}$, Alex W Hewitt ${ }^{265.266}$, Mika Kähönen ${ }^{262.268}$, Louis

63 Pérusse $^{2727269}$, Claude Bouchard ${ }^{270}$, Anke Tönjes ${ }^{211}$, Yii-Der Ida Chen ${ }^{83}$, Craig E Pennell2 ${ }^{221}$, Trevor

64 A Mori ${ }^{272}$, Wolfgang Lieb ${ }^{273}$, Andre Franke ${ }^{274}$, Claes Ohlsson ${ }^{215.275}$, Dan Mellström ${ }^{215.276}$, Yoon Shin

$65 \mathrm{Cho}^{214}$, Hyejin Lee ${ }^{277}$, Jian-Min Yuan ${ }^{27.279}$, Woon-Puay Koh ${ }^{280.281}$, Sang Youl Rhee ${ }^{282}$, Jeong-Taek

66 WoO $^{282}$, Iris M Heid ${ }^{11}$, Klaus J Stark", Martina E Zimmermann"1, Henry Völzke" ${ }^{283}$, Georg

67 Homuth $^{209}$, Michele K Evans ${ }^{284}$, Alan B Zonderman ${ }^{284}$, Ozren Polasek ${ }^{254,25}$, Gerard Pasterkamp ${ }^{206}$,

68 Imo E Hoefer ${ }^{206}$, Susan Redline ${ }^{204205}$, Katja Pahkala ${ }^{20220203286}$, Albertine J Oldehinkel ${ }^{287}$, Harold

69 Snieder $^{201}$, Ginevra Biino ${ }^{288}$, Reinhold Schmidt ${ }^{196}$, Helena Schmidt ${ }^{289}$, Stefania Bandinelli ${ }^{200}$,

70 George Dedoussis ${ }^{194}$, Thangavel Alphonse Thanaraj ${ }^{193}$, Patricia A Peyser ${ }^{58}$, Norihiro Kato ${ }^{177}$,

71 Matthias B Schulze ${ }^{191,192,291}$, Giorgia Girotto ${ }^{292,199}$, Carsten A Böger ${ }^{18,293,294}$, Bettina Jung ${ }^{189,293,294}$, Peter

72 K Joshi $^{168}$, David A Bennett ${ }^{187,188}$, Philip L De Jager ${ }^{295,296}$, Xiangfeng Lu ${ }^{107}$, Vasiliki Mamakou ${ }^{297.298}$,

73 Morris Brown ${ }^{299.88}$, Mark J Caulfield ${ }^{87.88}$, Patricia B Munroe ${ }^{87.88}$, Xiuqing Guo ${ }^{83}$, Marina Ciullo ${ }^{184,185}$,

74 Jost B. Jonas ${ }^{300.182 .301,302}$, Nilesh J Samani ${ }^{180.181}$, Jaakko Kaprio ${ }^{38}$, Päivi Pajukanta ${ }^{303}$, Teresa Tusié-

75 Luna $^{30,305}$, Carlos A Aguilar-Salinas ${ }^{306}$, Linda S Adair ${ }^{307,300}$, Sonny Augustin Bechayda ${ }^{3093.10}$, H. 
76 Janaka de Silva ${ }^{311}$, Ananda R Wickremasinghe ${ }^{312}$, Ronald M Krauss ${ }^{313}$, Jer-Yuarn Wu ${ }^{314}$, Wei

77 Zheng ${ }^{175}$, Anneke I den Hollander ${ }^{174}$, Dwaipayan Bharadwaj ${ }^{173,35}$, Adolfo Correa ${ }^{316}$, James G

78 Wilson $^{317}$, Lars Lind ${ }^{131}$, Chew-Kiat Heng ${ }^{319}$, Amanda E Nelson ${ }^{169320}$, Yvonne M Golightly ${ }^{16932131323232}$,

79 James F Wilson ${ }^{168,45}$, Brenda PenninX ${ }^{324325}$, Hyung-Lae Kim ${ }^{326}$, John Attia ${ }^{327,163}$, Rodney J Scott ${ }^{127,163}$,

80 D C Rao ${ }^{228}$, Donna K Arnett ${ }^{229}$, Mark Walker ${ }^{330}$, Laura J Scott ${ }^{13}$, Heikki A Koistinen ${ }^{39311.32}$, Giriraj

81 R Chandak ${ }^{161.162 .33}$, Josep M Mercader ${ }^{334335336,}$, Teresa Tusie-Luna ${ }^{337}$, Carlos Aguilar-Salinas ${ }^{338}$,

82 Clicerio Gonzalez Villalpando ${ }^{339}$, Lorena Orozco ${ }^{340}$, Myriam Fornage ${ }^{156.341}$, E Shyong Tai ${ }^{342.20}$, Rob

83 M van Dam ${ }^{80,32}$, Terho Lehtimäki ${ }^{282229}$, Nish Chaturvedi ${ }^{343}$, Mitsuhiro Yokota ${ }^{344}$, Jianjun Liu ${ }^{152}$,

84 Dermot F Reilly ${ }^{345}$, Amy Jayne McKnight ${ }^{147}$, Frank Kee ${ }^{147}$, Karl-Heinz Jöckel ${ }^{146}$, Mark I

85 McCarthy ${ }^{62.346}$, Colin NA Palmer ${ }^{347}$, Veronique Vitart ${ }^{145}$, Caroline Hayward ${ }^{145}$, Eleanor

86 Simonsick ${ }^{348}$, Cornelia M van Duijn ${ }^{139,140}$, Zi-Bing Jin ${ }^{349,30}$, Fan Lu $^{350}$, Haretsugu Hishigaki ${ }^{135}, \mathrm{Xu}$

87 Lin ${ }^{134}$, Winfried MärZ ${ }^{351,352,132}$, Vilmundur Gudnason ${ }^{131,353}$, Jean-Claude Tardif ${ }^{130}$, Guillaume

88 Lettre $^{130}$, Leen M t Hart ${ }^{354355,356}$, Petra JM Elders ${ }^{357}$, Daniel J Rader ${ }^{338}$, Scott M Damrauer ${ }^{359,360}$,

89 Meena Kumari ${ }^{361}$, Mika Kivimaki ${ }^{126}$, Pim van der Harst ${ }^{362}$, Tim D Spector ${ }^{122}$, Ruth J.F. Loos ${ }^{1213638,89}$,

90 Michael A Province ${ }^{114}$, Esteban J Parra ${ }^{119}$, Miguel Cruz ${ }^{364}$, Bruce M Psaty ${ }^{11,365,366,}$, Ivan

91 Brandslund ${ }^{117.367}$, Peter P Pramstaller ${ }^{116}$, Charles N Rotimi ${ }^{368}$, Kaare Christensen ${ }^{369}$, Samuli

92 Ripatti $^{383770,371}$, Elisabeth Widén ${ }^{38}$, Hakon Hakonarson ${ }^{32.373}$, Struan F.A. Grant ${ }^{373377,375}$, Lambertus

93 ALM Kiemeney ${ }^{376}$, Jacqueline de Graaf ${ }^{376}$, Markus Loeffler ${ }^{110,111}$, Florian Kronenberg ${ }^{109}$,

94 Dongfeng Gu ${ }^{107.37}$, Jeanette Erdmann ${ }^{378}$, Heribert Schunkert ${ }^{105,106}$, Paul W Franks ${ }^{108}$, Allan

95 Linneberg ${ }^{81.82}$, J. Wouter Jukema ${ }^{101.379}$, Amit V Khera ${ }^{380.381 .382 .383}$, Minna Männikkö ${ }^{384}$, Marjo-Riitta

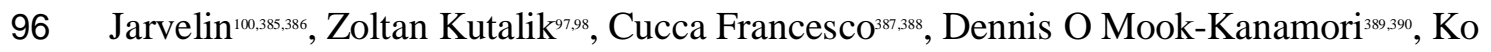

97 Willems van Dijk ${ }^{391,392.393}$, Hugh Watkins ${ }^{90.91}$, David P Strachan ${ }^{344}$, Niels Grarup ${ }^{89}$, Peter Sever ${ }^{395}$,

98 Neil Poulter ${ }^{336}$, Wayne Huey-Herng Sheu ${ }^{397,398}$, Jerome I Rotter ${ }^{33}$, Thomas M Dantoft ${ }^{81}$, Fredrik

99 Karpe $^{399,400}$, Matt J Neville ${ }^{399,400}$, Nicholas J Timpson ${ }^{35,79}$, Ching-Yu Cheng ${ }^{401,402}$, Tien-Yin Wong ${ }^{401,402}$,

100 Chiea Chuen Khor ${ }^{152}$, Hengtong Li $^{403}$, Charumathi Sabanayagam ${ }^{401,402}$, Annette Peters ${ }^{7,044,405}$, 
101 Christian Gieger ${ }^{7,677,405}$, Andrew T Hattersley ${ }^{406}$, Nancy L Pedersen ${ }^{407}$, Patrik KE Magnusson ${ }^{407}$,

102 Dorret I Boomsma ${ }^{72,408}$, Eco JC de Geus ${ }^{72,408}$, L Adrienne Cupples ${ }^{5,409}$, Joyce B.J. van Meurs ${ }^{7,140}$, 103 Arfan Ikram ${ }^{140}$, Mohsen Ghanbari ${ }^{140,410}$, Penny Gordon-Larsen ${ }^{307,308}$, Wei Huang ${ }^{411}$, Young Jin

$104 \mathrm{Kim}^{24}$, Yasuharu Tabara ${ }^{70}$, Nicholas J Wareham ${ }^{68}$, Claudia Langenberg ${ }^{68}$, Eleftheria

105 Zeggini $^{218,47,412}$, Jaakko Tuomilehto ${ }^{39,413,44}$, Johanna Kuusisto ${ }^{415}$, Markku Laakso ${ }^{415}$, Erik

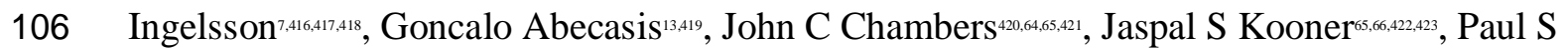

107 de Vries ${ }^{63}$, Alanna C Morrison ${ }^{63}$, Scott Hazelhurst ${ }^{61.24}$, Michèle Ramsay ${ }^{61}$, Kari E. North ${ }^{425}$,

108 Martha Daviglus ${ }^{426}$, Peter Kraft ${ }^{59,427}$, Nicholas G Martin ${ }^{428}$, John B Whitfield ${ }^{228}$, Shahid Abbas ${ }^{429}$,

109 Danish Saleheen ${ }^{55,430,431}$, Robin G Walters ${ }^{5,3,4,432}$, Michael V Holmes ${ }^{5,3,54,433}$, Corri Black ${ }^{434}$, Blair H

110 Smith $^{435}$, Aris Baras ${ }^{419}$, Anne E Justice ${ }^{436}$, Julie E Buring ${ }^{50.437}$, Paul M Ridker ${ }^{50.437}$, Daniel I

111 Chasman $^{50.437}$, Charles Kooperberg ${ }^{49}$, Gen Tamiya ${ }^{46}$, Masayuki Yamamoto ${ }^{46}$, David A van Heel ${ }^{48}$,

112 Richard C Trembath ${ }^{438}$, Wei-Qi Wei ${ }^{439}$, Gail P Jarvik ${ }^{40}$, Bahram Namjou ${ }^{411}$, M. Geoffrey

113 Hayes $^{42,443,44}$, Marylyn D Ritchie ${ }^{40}$, Pekka Jousilahti $1^{39}$, Veikko Salomaa ${ }^{39}$, Kristian Hveem ${ }^{34,45,46}$,

114 Bjørn Olav Åsvold ${ }^{3,445,44}$, Michiaki Kubo ${ }^{448}$, Yoichiro Kamatani ${ }^{25.49}$, Yukinori Okada ${ }^{27,25,450,451}$,

115 Yoshinori Murakami ${ }^{452}$, Bong-Jo Kim ${ }^{453}$, Unnur Thorsteinsdottir ${ }^{21,444}$, Kari Stefansson ${ }^{2.1454}$, Jifeng

116 Zhangs, Y Eugene Chen ${ }^{3}$, Yuk-Lam Ho ${ }^{455}$, Julie A Lynch ${ }^{456,457}$, Daniel Rader ${ }^{458}$, Philip S Tsao ${ }^{6,74,49}$,

117 Kyong-Mi Chang ${ }^{40,458}$, Kelly ChO ${ }^{455,461}$, Christopher J O'Donnell ${ }^{455,461}$, John M Gaziano ${ }^{455,461}$, Peter

118 Wilson $^{17,462}$, Karen L Mohlke ${ }^{178}$, Timothy M Frayling ${ }^{74}$, Joel N Hirschhorn ${ }^{43,10,664}$, Sekar

119 Kathiresan ${ }^{45.5831 .383}$, Michael Boehnke ${ }^{13}$, Million Veterans Program, Global Lipids Genetics

120 Consortium, Struan Grant ${ }^{1.374}$, Pradeep Natarajan ${ }^{46,46,2.20,468}$, Yan V Sun ${ }^{1.17}$, Andrew P Morris ${ }^{469}$,

121 Panos Deloukas ${ }^{4,470}$, Gina Peloso ${ }^{471}$, Themistocles L Assimes ${ }^{6,7,459}$, Cristen J Willer ${ }^{3,472,145}$, Xiang

122

Zhu $^{473,47,475,476,}$ Christopher D Brown ${ }^{1}$ 
$124{ }^{1}$ Department of Genetics, Perelman School of Medicine, University of Pennsylvania,

125 Philadelphia, PA 19104, USA, ${ }^{2}$ Department of Genetics, Stanford University School of

126 Medicine, Stanford, CA, USA, ${ }^{3}$ Department of Internal Medicine, Division of Cardiology,

127 University of Michigan, Ann Arbor, MI, 48109, USA, ${ }^{4}$ William Harvey Research Institute,

128 Barts and the London School of Medicine and Dentistry, Queen Mary University of London,

129 Charterhouse square, EC1M 6BQ, UK, ${ }^{5}$ Department of Biostatistics, Boston University

130 School of Public Health, 801 Massachusetts Ave, Boston, MA 02118, USA, ${ }^{\circ V A}$ Palo Alto

131 Health Care Systems, Palo Alto, California, USA, 'Department of Medicine, Division of

132 Cardiovascular Medicine, Stanford University School of Medicine, Stanford, CA 94305,

133 USA, ${ }^{8}$ Department of Pathology and Laboratory Medicine, University of Pennsylvania,

134 Philadelphia, PA, USA, ${ }^{\circledR}$ Endocrinology, Boston Childrens Hospital, Boston 02115 MA,USA,

135 ${ }^{10}$ Medical and Population Genetics, Broad Institute, 75 Ames street, Cambridge, MA

136 02142,USA, "Department of Genetic Epidemiology, University of Regensburg, Regensburg,

137 Germany, ${ }^{2} \mathrm{McD}$ Donnell Genome Institute and Department of Medicine, Washington

138 University, St. Louis, MO, 63108, USA, ${ }^{13}$ Department of Biostatistics, Center for Statistical

139 Genetics, University of Michigan, Ann Arbor, MI, USA, ${ }^{14}$ Department of Computational

140 Medicine and Bioinformatics, University of Michigan, Ann Arbor, MI, USA, ${ }^{15}$ Clinical

141 Pharmacology, William Harvey Research Institute, Barts and The London School of

142 Medicine and Dentistry, Queen Mary University of London, London, EC1M 6BQ UK,

$143{ }^{16}$ Department of Epidemiology, Emory University Rollins School of Public Health, Atlanta,

144 Georgia, USA, ${ }^{17}$ Atlanta VA Health Care System, Decatur, GA, USA, ${ }^{18}$ Malcolm Randall VA

145 Medical Center, Gainesville, FL, USA, ${ }^{19}$ Division of Vascular Surgery and Endovascular

146 Therapy, University of Florida College of Medicine, Gainesville, FL, USA, ${ }^{20}$ Program in

147 Medical and Population Genetics, Broad Institute of Harvard and MIT, Cambridge, MA,

148 USA, ${ }^{21}$ deCODE genetics/Amgen, Inc. Sturlugata 8, Reykjavik, 102, Iceland, ${ }^{22}$ School of 
149 Engineering and Natural Sciences, University of Iceland, Sæmundargötu 2, Reykjavik, 102,

150 Iceland, ${ }^{23}$ Department of Clinical Biochemistry, Landspitali - National University Hospital of

151 Iceland, Hringbraut, Reykjavik, 101, Iceland, ${ }^{24}$ Division of Genome Science, Department of

152 Precision Medicine, National Institute of Health, Chungcheongbuk-do, South Korea,

$153{ }^{25}$ Laboratory for Statistical Analysis, RIKEN Center for Integrative Medical Sciences,

154 Yokohama, Japan, ${ }^{26}$ Department of Ophthalmology, Graduate School of Medical Sciences,

155 Kyushu University, Fukuoka, Japan, ${ }^{2}$ Department of Statistical Genetics, Osaka University

156 Graduate School of Medicine, Osaka, Japan, ${ }^{28}$ Laboratory for Statistical Analysis, RIKEN

157 Center for Integrative Medical Sciences, ${ }^{2}$ Department of Allergy and Rheumatology,

158 Graduate School of Medicine, The University of Tokyo, Tokyo, Japan, ${ }^{30}$ Laboratory for

159 Statistical and Translational Genetics, RIKEN Center for Integrative Medical Sciences,

160 Yokohama, Japan, ${ }^{3}$ Program in Medical and Population Genetics, Broad Institute of MIT and

161 Harvard, Cambridge, MA, USA., ${ }^{32}$ Department of Biomedical Informatics, Harvard Medical

162 School, Boston, MA, USA, ${ }^{33}$ Analytic and Translational Genetics Unit, Massachusetts

163 General Hospital, Boston, Massachusetts, USA, ${ }^{34}$ K.G. Jebsen Center for Genetic

164 Epidemiology, Department of Public Health and Nursing, NTNU, Norwegian University of

165 Science and Technology, Trondheim, Norway, ${ }^{35}$ MRC Integrative Epidemiology Unit (IEU),

166 Bristol Medical School, University of Bristol, Oakfield House, Oakfield Grove, Bristol, BS8

167 2BN, UK, ${ }^{36}$ Clinic of Medicine, St. Olavs Hospital, Trondheim University Hospital,

168 Trondheim, Norway, ${ }^{3}$ Division of Medicine and Laboratory Sciences, University of

169 Oslo,Norway, ${ }^{38}$ Institute for Molecular Medicine Finland (FIMM), HiLIFE, University of

170 Helsinki, Tukholmankatu 8, 00014 Helsinki, Finland, ${ }^{39}$ Department of Public Health and

171 Welfare, Finnish Institute for Health and Welfare, Helsinki, Finland, ${ }^{40}$ Department of

172 Genetics, Institute for Biomedical Informatics, University of Pennsylvania, Perelman School

173 of Medicine, Philadelphia, PA 19104, USA, ${ }^{4}$ Center for Genetic Medicine, Northwestern 
174 University, Feinberg School of Medicine, Chicago, IL 60611, USA, ${ }^{4}$ Department of

175 Medicine (Medical Genetics), University of Washington, WA, USA, ${ }^{43}$ Division of Biomedical

176 Informatics, Cincinnati Children's Hospital Medical Center, OH, USA, ${ }^{4}$ Division of Clinical

177 Pharmacology, Department of Medicine, Vanderbilt University Medical Center, Nashville,

178 TN, USA, ${ }^{45}$ Department of Cardiovascular Medicine and the Gonda Vascular Center, Mayo

179 Clinic, Rochester, MN, USA, ${ }^{4}$ Tohoku Medical Megabank Organization, Tohoku University,

180 Sendai 980-8573, Japan, ${ }^{47}$ Wellcome Trust Sanger Institute, Hinxton, CB10 1SA, UK,

$181{ }^{48}$ Blizard Institute, Barts and the London School of Medicine and Dentistry, Queen Mary

182 University of London, London, UK, ${ }^{4}$ Fred Hutchinson Cancer Research Center, Division of

183 Public Health Sciences, Seattle WA 9810, USA, ${ }^{50}$ Division of Preventive Medicine, Brigham

184 and Women's Hospital, Boston, MA 02215, USA, ${ }^{5}$ Centre for Genomic and Experimental

185 Medicine, Institute of Genetics and Cancer, University of Edinburgh, Western General

186 Hospital, Edinburgh EH4 2XU, United Kingdom, ${ }^{52}$ Usher Institute, The University of

187 Edinburgh, Nine, Edinburgh Bioquarter, 9 Little France Road, Edinburgh, EH16 4UX, UK,

$188{ }^{33}$ Clinical Trial Service Unit and Epidemiological Studies Unit, Nuffield Department of

189 Population Health, University of Oxford, Oxford OX3 7LF, UK, ${ }^{54}$ Medical Research Council

190 Population Health Research Unit, Nuffield Department of Population Health, University of

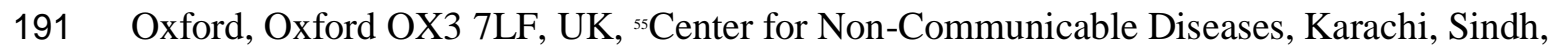

192 Pakistan, ${ }^{56}$ Department of Population Medicine, Qatar University College of Medicine, QU

193 Health, Doha, Qatar, ${ }^{57}$ Survey Research Center, Institute for Social Research, University of

194 Michigan, Ann Arbor, MI, 48104, USA, ${ }^{58}$ Department of Epidemiology, School of Public

195 Health, University of Michigan, Ann Arbor, MI, 48109, USA, ${ }^{5}$ Program in Genetic

196 Epidemiology and Statistical Genetics, Department of Epidemiology, Harvard T.H. Chan

197 School of Public Health, 677 Huntington Avenue, Boston, MA, 02115, USA, ${ }^{60}$ Department of

198 Epidemiology, Gillings School of Global Public Health, University of North Carolina at 
199 Chapel Hill, NC USA, ${ }^{6}$ Sydney Brenner Institute for Molecular Bioscience, Faculty of Health

200 Sciences, University of the Witwatersrand, Johannesburg, South Africa, ${ }^{6}$ Wellcome Centre

201 for Human Genetics, University of Oxford, UK, ${ }^{63}$ Human Genetics Center, Department of

202 Epidemiology, Human Genetics, and Environmental Sciences, School of Public Health, The

203 University of Texas Health Science Center at Houston, Houston, Texas, 77030, USA,

$204{ }^{64}$ Department of Epidemiology and Biostatistics, Imperial College London, London W2 1PG,

205 UK, ${ }^{65}$ Department of Cardiology, Ealing Hospital, London North West University Healthcare

206 NHS Trust, Middlesex UB1 3HW, UK, ${ }^{6}$ Imperial College Healthcare NHS Trust, London

207 W12 0HS, UK, ${ }^{6}$ Department of Medical Sciences, Molecular Epidemiology and Science for

208 Life Laboratory, Uppsala University, Uppsala, Sweden, ${ }^{6}$ MRC Epidemiology Unit,

209 University of Cambridge School of Clinical Medicine, Cambridge, CB2 0QQ, UK,

$210{ }^{69}$ Cardiovascular Epidemiology Unit, Department of Public Health and Primary Care,

211 University of Cambridge, Strangeways Research Laboratory, Wort's Causeway, Cambridge

212 CB1 8RN, UK, ${ }^{70}$ Center for Genomic Medicine, Kyoto University Graduate School of

213 Medicine, Kyoto, Japan, ${ }^{7}$ Department of Internal Medicine, Erasmus MC, University Medical

214 Center Rotterdam, the Netherlands, ${ }^{22}$ Department of Biological Psychology, Vrije Universiteit

215 Amsterdam, The Netherlands, ${ }^{73}$ Amsterdam Public Health Research Institute, Amsterdam

216 UMC, the Netherlands, ${ }^{74}$ Genetics of Complex Traits, University of Exeter Medical School,

217 University of Exeter, Exeter, EX2 5DW, UK, ${ }^{75}$ Department of Clinical Acupuncture and

218 Moxibustion, Nanjing University of Chinese Medicine, Nanjing, Jiangsu 210029, China,

$219{ }^{7}$ Research Unit of Molecular Epidemiology, Helmholtz Zentrum München, German Research

220 Center for Environmental Health, Neuherberg, Germany, ${ }^{7}$ Institute of Epidemiology,

221 Helmholtz Zentrum München, German Research Center for Environmental Health,

222 Neuherberg, Germany, ${ }^{78}$ Bristol Dental School, University of Bristol, Lower Maudlin Street,

223 Bristol BS1 2LY, United Kingdom, ${ }^{79}$ Population Health Sciences, Bristol Medical School, 
224 University of Bristol, Oakfield Grove, Bristol, BS8 2BN, United Kingdom, ${ }^{80}$ Saw Swee Hock

225 School of Public Health, National University of Singapore and National University Health

226 System, 117549, Singapore, ${ }^{8}$ Center for Clinical Research and Prevention, Bispebjerg and

227 Frederiksberg Hospital, Copenhagen, Denmark, ${ }^{82}$ Department of Clinical Medicine, Faculty of

228 Health and Medical Sciences, University of Copenhagen, Copenhagen, Denmark, ${ }^{83}$ The

229 Institute for Translational Genomics and Population Sciences, Department of Pediatrics,

230 Lundquist Institute for Biomedical Innovations (Formerly LABioMed) at Harbor-UCLA

231 Medical Center, Torrance, CA 90502, USA, ${ }^{8}$ Center for Public Health Genomics, University

232 of Virginia, Charlottesville, VA 22903 USA, ${ }^{85}$ Department of Medical Research, Taichung

233 Veterans General Hospital, Taichung, Taiwan; No. 1650, Sec. 4, Taiwan Boulevard,

234 Taichung City 40705, Taiwan, ${ }^{8}$ Institute of Population Health Sciences, National Health

235 Research Institutes, 35 Keyan Road, Zhunan Town, Miaoli County 350, Taiwan, ROC,

$236{ }^{87}$ William Harvey Research Institute, Barts and The London School of Medicine and

237 Dentistry, Queen Mary University of London, John Vane Science Centre, Charterhouse

238 Square, London, EC1M 6BQ, UK, ${ }^{88}$ NIHR Barts Cardiovascular Biomedical Research Centre,

239 Barts and The London School of Medicine and Dentistry, Queen Mary University of London,

240 London, EC1M 6BQ, UK, ${ }^{8}$ Novo Nordisk Foundation Center for Basic Metabolic Research,

241 Faculty of Health and Medical Sciences, University of Copenhagen, Copenhagen, Denmark,

$242{ }^{9}$ Division of Cardiovascular Medicine, Radcliffe Department of Medicine, John Radcliffe

243 Hospital, University of Oxford, Oxford. UK. OX3 9DU, ${ }^{9}$ Wellcome Centre for Human

244 Genetics, University of Oxford, Oxford. UK. OX3 7BN, ${ }^{2}$ Unit of Genomics of Complex

245 Diseases. Sant Pau Biomedical Research Institute (IIB Sant Pau), Barcelona, Spain,

$246{ }_{93}$ Cardiovascular Medicine Unit, Department of Medicine, Karolinska Institutet, Center for

247 Molecular Medicine, Karolinska University Hospital, Stockholm, Sweden, ${ }^{94}$ Department of

248 Internal Medicine, Section of Gerontology and Geriatrics, Leiden University Medical Center, 
249 Leiden, the Netherlands, ${ }^{95}$ Istituto di Ricerca Genetica e Biomedica, Consiglio Nazionale delle

250 Ricerche, Italy, ${ }^{9}$ Dipartimento di Scienze Biomediche, Università degli Studi di Sassari,

251 Sardinia, Italy, ${ }^{9}$ University Center for Primary Care and Public Health, University of

252 Lausanne, Rte de Berne 113, Lausanne, 1010, Switzerland, ${ }^{9}$ Swiss Institute of

253 Bioinformatics, Lausanne, 1015, Switzerland, ${ }^{90}$ Department of Medicine, Internal Medicine,

254 Lausanne University Hospital and University of Lausanne, Rue du Bugnon 46, Lausanne,

255 1011, Switzerland, ${ }^{10}$ Department of Epidemiology and Biostatistics, MRC-PHE Centre for

256 Environment and Health, School of Public Health, Imperial College London, London, UK,

$257{ }^{101}$ Dept of Cardiology, Leiden University Medical Center, Leiden, the Netherlands, ${ }^{102}$ Dept of

258 Internal Medicine, Section of Gerontology and Geriatrics, Leiden university Medical Center,

259 Leiden, the Netherlands, ${ }^{103}$ BHF Glasgow Cardiovascular Research Centre, Faculty of

260 Medicine, Glasgow, United Kingdom, ${ }^{104}$ Institute for Cardiogenetics, University of Lübeck,

261 DZHK (German Research Centre for Cardiovascular Research), partner site

262 Hamburg/Lübeck/Kiel, University Heart Center Lübeck, Lübeck and Charité - University

263 Medicine Berlin, corporate member of Freie Universität Berlin, Humboldt-Universität zu

264 Berlin, and Berlin Institute of Health, Institute for Dental and Craniofacial Sciences,

265 Department of Periodontology and Synoptic Dentistry, Berlin, Germany, ${ }^{105}$ Deutsches

266 Herzzentrum München, Klinik für Herz- und Kreislauferkrankungen, Technische Universität

267 München,Munich, Germany., ${ }^{10}$ Deutsches Zentrum für Herz-Kreislauf-Forschung (DZHK)

268 e.V., partner site Munich Heart Alliance,Munich, Germany., ${ }^{100}$ Key Laboratory of

269 Cardiovascular Epidemiology \& Department of Epidemiology, State Key Laboratory of

270 Cardiovascular Disease, Fuwai Hospital, National Center for Cardiovascular Diseases,

271 Chinese Academy of Medical Sciences and Peking Union Medical College, Beijing 100037,

272 China, ${ }^{108}$ Lund University Diabetes Centre, Malmö, Sweden, ${ }^{10}$ Institute of Genetic

273 Epidemiology, Department of Genetics and Pharmacology, Medical University of Innsbruck, 
274 Innsbruck, Austria and German Chronic Kidney Disease Study, Austria, ${ }^{11}$ Institute for

275 Medical Informatics, Statistics and Epidemiology, University of Leipzig, Haertelstrasse 16-

276 18, 04107 Leipzig, Germany, "'LIFE Research Centre for Civilization Diseases, University of

277 Leipzig, Philipp-Rosenthal-Straße 27, 04103 Leipzig, Germany, ${ }^{112}$ Radboud university

278 medical center, Radboud Institute for Health Sciences, Nijmegen, the Netherlands,

$279{ }^{113}$ Quantinuum Research LLC, Wayne, PA, 19087 USA, ${ }^{114}$ Division of Statistical Genomics,

280 Department of Genetics; Washington University School of Medicine; St. Louis, MO, USA,

$281 \quad{ }^{115}$ Department of Epidemiology; University of Pittsburgh; Pittsburgh, PA, USA, ${ }^{116}$ Institute for

282 Biomedicine, Eurac Research, Affiliated Institute of the University of Lübeck, Via Galvani

283 31, 39100, Bolzano, Italy, "'Department of Clinical Biochemistry, Lillebaelt Hospital, Vejle,

284 Denmark, ${ }^{118}$ Cardiovascular Health Research Unit, Department of Medicine, University of

285 Washington, Seattle, 98101, USA, ${ }^{119}$ Department of Anthropology, University of Toronto at

286 Mississauga, Mississauga, ON L5L 1C6, Canada, ${ }^{120}$ Department of Computer Science,

287 University of Toronto, Toronto, ON M5S 2E4, Canada, ${ }^{12}$ The Charles Bronfman Institute for

288 Personalized Medicine, Icahn School of Medicine at Mount Sinai, New York, NY, USA,

$289{ }^{122}$ Department of Twin Research and Genetic Epidemiology, King's College London, London

290 SE1 7EH, UK, ${ }^{123}$ NIHR Biomedical Research Centre at Guy's and St Thomas' Foundation

291 Trust, London SE1 9RT, UK, ${ }^{124}$ Department of Cardiology, University of Groningen,

292 University Medical Center Groningen, 9700RB Groningen, the Netherlands, ${ }^{12}$ Institute of

293 Cardiovascular Sciences, University College London, Gower Street, WC1E 6BT London,

294 UK, ${ }^{126}$ Department of Epidemiology and Public Health, University College London, 1-19

295 Torrington Place, WC1E 6BT London, United Kingdom, ${ }^{12}$ Department of Surgery, University

296 of Pennsylvania, Philadelphia, PA, ${ }^{128}$ Amsterdam UMC, Department of Epidemiology and

297 Data Science, Amsterdam Public Health Research Institute, Amsterdam, 1081HV, the

298 Netherlands, ${ }^{122}$ Leiden University Medical Center, Department of Cell and Chemical Biology, 
299 Leiden, 2333ZA, the Netherlands, ${ }^{130}$ Montreal Heart Institute, Université de Montréal, 5000

300 Belanger street, Montreal, PQ, Canada H1T1C8, ${ }^{13}$ Icelandic Heart Association, 201

301 Kopavogur, Iceland, ${ }^{132}$ Vth Department of Medicine, Medical Faculty Mannheim, Heidelberg

302 University, 68167 Mannheim, Germany, ${ }^{133}$ SYNLAB MVZ Humangenetik Mannheim GmbH,

30368163 Mannheim, Germany, ${ }^{134}$ Shanghai Institute of Nutrition and Health, University of

304 Chinese Academy of Sciences, Chinese Academy of Sciences, Shanghai, China, ${ }^{135}$ Biomedical

305 Technology Research Center, Tokushima Research Institute, Otsuka Pharmaceutical Co.,

306 Ltd., Tokushima, Japan, ${ }^{136}$ School of Life Sciences, Westlake University, Hangzhou, Zhejiang

307 310024, China, ${ }^{137}$ Westlake Laboratory of Life Sciences and Biomedicine, Hangzhou,

308 Zhejiang 310024, China, ${ }^{138}$ Institute for Molecular Bioscience, The University of Queensland,

309 Brisbane, Queensland 4072, Australia, ${ }^{139}$ Nuffield Department of Population Health,

310 University of Oxford, Oxford, United Kingdom, ${ }^{140}$ Department of Epidemiology, Erasmus

311 MC, University Medical Center Rotterdam, the Netherlands, ${ }^{14}$ Department of Epidemiology,

312 Erasmus MC, University Medical Center, Rotterdam, the Netherlands, ${ }^{142}$ Section of Statistical

313 Multi-omics, Department of Clinical and Experimental research, University of Surrey,

314 Guildford, Surrey, UK, ${ }^{143}$ Laboratory of Neurogenetics, National Institute on Aging, NIH,

315 Bethesda MD, USA, ${ }^{14}$ Data Tecnica International, Glen Echo MD, USA, ${ }^{14}$ MRC Human

316 Genetics Unit, Institute of Genetics and Cancer, University of Edinburgh, Western General

317 Hospital, Crewe Road, Edinburgh, EH4 2XU, Scotland, ${ }^{14}$ Institute for Medical Informatics,

318 Biometrie and Epidemiology, University of Duisburg-Essen, Essen, Germany, ${ }^{147}$ Centre for

319 Public Health, Queen's University of Belfast, Northern Ireland, ${ }^{148}$ Genomic Oncology Area,

320 GENYO, Centre for Genomics and Oncological Research: Pfizer-University of Granada-

321 Andalusian Regional Government, Granada, Spain, ${ }^{14}$ Hematology Department, Hospital

322 Universitario Virgen de las Nieves, Granada, Spain, ${ }^{150}$ Instituto de Investigación Biosanitaria

323 de Granada (ibs.GRANADA), Granada, Spain, ${ }^{15}$ Department of Epidemiology and 
324 Biostatistics, School of Public Health, Tongji Medical College, Huazhong University of

325 Science and Technology, Wuhan, China, ${ }^{152}$ Genome Institute of Singapore, Agency for

326 Science, Technology and Research, Singapore, ${ }^{153}$ Public Health Informatics Unit, Department

327 of Integrated Health Sciences, Nagoya University Graduate School of Medicine, Nagoya,

328 461-8673, Japan, ${ }^{15}$ University of Alabama at Birmingham, Epidemiology, School of Public

329 Health, AL, USA, ${ }^{155}$ Tampere Centre for Skills Training and Simulation, Faculty of Medicine

330 and Health Technology, Tampere University, Tampere, Finland, ${ }^{156}$ Brown Foundation

331 Institute of Molecular Medicine, McGovern Medical School, University of Texas Health

332 Science Center at Houston, Houston TX 77030, USA, ${ }^{15}$ CONACYT, Instituto Nacional de

333 Ciencias Médicas y Nutrición Salvador Zubirán, Ciudad de Mexico, Mexico, ${ }^{158}$ Departamento

334 de Genómica Computacional, Instituto Nacional de Medicina Genómica, Ciudad de Mexico,

335 Mexico, ${ }^{150}$ Center for diabetes research, University of Bergen, Bergen, Norway, ${ }^{160}$ Lund

336 University Diabetes Center, Lunds University, Malmö, Sweden, ${ }^{16}$ Genomic Research on

337 Complex diseases (GRC Group), CSIR-Centre for Cellular and Molecular Biology,

338 Hyderabad, Telangana, India, ${ }^{162}$ Academy of Scientific and Innovative Research (AcSIR),

339 New Delhi, India, ${ }^{163}$ Hunter Medical Research Institute, Newcastle, Australia, ${ }^{164}$ Medical

340 Research Institute, Kangbuk Samsung Hospital, Sungkyunkwan University School of

341 Medicine, Seoul, 03181, Korea, ${ }^{165}$ Department of Clinical Research Design \& Evaluation,

342 SAIHST, Sungkyunkwan University, Seoul, 06355, Korea, ${ }^{16}$ Center for Cohort Studies, Total

343 Healthcare Center, Kangbuk Samsung Hospital, Sungkyunkwan University School of

344 Medicine, Seoul, 04514, Korea, ${ }^{167}$ Department of Occupational and Environmental Medicine,

345 Kangbuk Samsung Hospital, Sungkyunkwan University School of Medicine, Seoul, 03181,

346 Korea, ${ }^{16}$ Centre for Global Health Research, Usher Institute, University of Edinburgh, Teviot

347 Place, Edinburgh, EH8 9AG, Scotland, ${ }^{16}$ Thurston Arthritis Research Center, University of

348 North Carolina, Chapel Hill, North Carolina, USA, ${ }^{17}$ Health Services and Systems Research, 
349 Duke-NUS Medical School, 169857, Singapore, ${ }^{17}$ Division of Biomedical Informatics and

350 Personalized Medicine, Department of Medicine, Anschutz Medical Campus, University of

351 Colorado, Denver, Aurora, CO 80045, USA, ${ }^{17}$ Genomics and Molecular Medicine Unit,

352 CSIR-Institute of Genomics and Integrative Biology, New Delhi - 110020, India, ${ }^{173}$ Academy

353 of Scientific and Innovative Research, CSIR-Human Resource Development Centre,

354 Ghaziabad, Uttar Pradesh, India, ${ }^{17}$ Departments of Ophthalmology and Human Genetics,

355 Radboud University Nijmegen Medical Center, Philips van Leydenlaan 15, Nijmegen, 6525

356 EX, the Netherlands, ${ }^{175}$ Vanderbilt Epidemiology Center, Division of Epidemiology,

357 Vanderbilt University Medical Center, USA, ${ }^{176}$ Department of Pediatrics, University of

358 California San Francisco, Oakland, CA 94609 USA, ${ }^{n 7}$ National Center for Global Health and

359 Medicine, Tokyo, 1628655, Japan, ${ }^{178}$ Department of Genetics, University of North Carolina,

360 Chapel Hill, NC 27599 USA, ${ }^{179}$ Department of Biostatistics and Epidemiology, University of

361 Massachusetts-Amherst, Amherst, MA 01003 USA, ${ }^{180}$ Department of Cardiovascular

362 Sciences, University of Leicester, Leicester, UK, ${ }^{18}$ NIHR Leicester Biomedical Research

363 Centre, Glenfield Hospital, Leicester, UK, ${ }^{182}$ Beijing Institute of Ophthalmology, Beijing Key

364 Laboratory of Ophthalmology and Visual Sciences, Beijing Tongren Eye Center, Beijing

365 Tongren Hospital, Capital Medical University, 17 Hougou Lane, Chong Wen Men, Beijing,

366100005 , China, ${ }^{183}$ Beijing Tongren Eye Center, Beijing Tongren Hospital, Capital Medical

367 University, 1 Dong Jiao Min Xiang, Dong Cheng District, Beijing, 100730, China, ${ }^{18}$ Institute

368 of Genetics and Biophysics "Adriano Buzzati-Traverso" - CNR, Naples, Italy, ${ }^{185}$ IRCCS

369 Neuromed, Pozzilli, Isernia, Italy, ${ }^{186}$ Division of Biostatistics, Washington University, St.

370 Louis, MO 63110, USA, ${ }^{18}$ Rush Alzheimer's Disease Center, Rush University Medical

371 Center, IL, USA, ${ }^{18}$ Department of Neurological Sciences, Rush University Medical Center,

372 IL, USA, ${ }^{18}$ Dept of Nephrology, University Hospital Regensburg, Regensburg, Germany,

$373{ }^{190}$ Institute for Maternal and Child Health-IRCCS, Burlo Garofolo, 34127 Trieste, Italy, 
$374{ }^{191}$ Department of Molecular Epidemiology, German Institute of Human Nutrition Potsdam-

375 Rehbruecke, Nuthetal, Germany, ${ }^{192}$ German Center for Diabetes Research (DZD), München-

376 Neuherberg, Germany, ${ }^{193}$ Department of Genetics and Bioinformatics, Dasman Diabetes

377 Institute, Kuwait, ${ }^{194}$ Department of Nutrition and Dietetics, School of Health Science and

378 Education, Harokopio University of Athens, Athens, Greece, ${ }^{195}$ Department of Population

379 Science and Experimental Medicine, University College London, London, UK, ${ }^{196}$ Clinical

380 Division of Neurogeriatrics, Department of Neurology, Medical University of Graz, Graz,

381 Austria, ${ }^{197}$ Institute for Medical Informatics, Statistics and Documentation, Medical University

382 of Graz, Graz, Austria, ${ }^{198}$ Massachusetts General Hospital Cancer Center, Charlestown, MA

383 02129, USA, ${ }^{199}$ Institute for Maternal and Child Health, IRCCS Burlo Garofolo, Trieste, Italy,

$384{ }^{200}$ Institute of Genetic and Biomedical Research, National Research Council of Italy, UOS of

385 Sassari, Sassari, Italy, ${ }^{201}$ Department of Epidemiology, University of Groningen, University

386 Medical Center Groningen, Groningen, 9700 RB, the Netherlands, ${ }^{20}$ Research Centre of

387 Applied and Preventive Cardiovascular Medicine, University of Turku, Turku, Finland,

$388{ }^{203}$ Centre for Population Health Research, University of Turku and Turku University Hospital,

389 Turku, Finland, ${ }^{204}$ Sleep Medicine and Circadian Disorders, Brigham and Women's Hospital,

390 Boston, Massachusetts 02115, USA, ${ }^{205}$ Division of Sleep Medicine, Harvard Medical School,

391 Boston, Massachusetts 02115, USA, ${ }^{206}$ Central Diagnostics Laboratory, Division Laboratories,

392 Pharmacy, and Biomedical genetics, University Medical Center Utrecht, Utrecht University,

393 Utrecht, the Netherlands, ${ }^{20}$ Laboratory of Epidemiology and Population Science National

394 Institute on Aging Intramural Research Program, NIH, USA, ${ }^{208}$ Fels Cancer Institute for

395 Personalized Medicine, Temple University Lewis Katz School of Medicine, Philadelphia,

396 PA, USA, ${ }^{200}$ Interfaculty Institute for Genetics and Functional Genomics, Department of

397 Functional Genomics, University of Greifswald and University Medicine Greifswald,

398 Greifswald, Germany, ${ }^{210}$ Center for Research on Genomics and Global Health, National 
399 Human Genome Research Institute, National Institutes of Health, 12 South Drive, Room 400 4047, Bethesda, MD, 20892, USA, ${ }^{21}$ Oneomics. co. 1td. 2F, Soonchunhyang Mirai Medical

401 Center 173, Buheuyng-ro, Bucheon-si Gyeonggi-do, 14585, Korea, ${ }^{212}$ Department of Clinical

402 Biochemistry and Immunology, Hospital of Southern Jutland, Kresten Philipsens Vej 15,

4036200 Aabenraa, Denmark, ${ }^{213}$ Department of Clinical Biochemistry, Lillebaelt Hospital,

404 Kolding, Denmark, ${ }^{214}$ Department of Biomedical Science, Hallym University, Chuncheon,

405 Gangwon-do 24252, Korea, ${ }^{215}$ Centre for Bone and Arthritis Research, Department of Internal

406 Medicine and Clinical Nutrition, Institute of Medicine, Sahlgrenska Academy, University of

407 Gothenburg, Gothenburg, Sweden, ${ }^{216}$ Bioinformatics Core Facility, Sahlgrenska Academy,

408 University of Gothenburg, Gothenburg, Sweden, ${ }^{21}$ Institute of Medical Informatics and

409 Statistics, Kiel University, Kiel, Germany, ${ }^{218}$ Institute of Translational Genomics, Helmholtz

410 Zentrum München - German Research Center for Environmental Health, Neuherberg,

411 Germany, ${ }^{21}$ Wellcome Centre for Human Genetics, University of Oxford, Oxford, UK,

$412{ }^{220}$ Oxford Centre for Diabetes Endocrinology and Metabolism, Oxford, UK, ${ }^{221}$ School of

413 Medicine and Public Health, Faculty of Medicine and Health, University of Newcastle,

414 Newcastle, New South Wales, 2308, Australia, ${ }^{22}$ Center for Geriatrics and Gerontology,

415 Division of Endocrinology and Metabolism, Department of Internal Medicine, Taichung

416 Veterans General Hospital, Taichung, Taiwan, ${ }^{223}$ School of Medicine, National Yang-Ming

417 University, Taipei, Taiwan, ${ }^{224}$ School of Medicine, National Defense Medical Center, Taipei,

418 Taiwan, ${ }^{225}$ Division of Endocrinology and Metabolism, Department of Internal Medicine,

419 Taichung Veterans General Hospital, Taichung, Taiwan, ${ }^{226}$ Department of Medicine, School

420 of Medicine, National Yang-Ming University, Taipei, Taiwan, ${ }^{22}$ Department of Kinesiology,

421 Université Laval, Québec, Canada, ${ }^{228}$ Department of Clinical Chemistry, Fimlab Laboratories,

422 Tampere 33520, Finland, ${ }^{229}$ Department of Clinical Chemistry, Finnish Cardiovascular

423 Research Center - Tampere, Faculty of Medicine and Health Technology, Tampere 
424 University, Tampere 33014, Finland, ${ }^{230}$ Department of Cardiology, Heart Center, Tampere

425 University Hospital, Tampere 33521, Finland, ${ }^{23}$ Department of Cardiology, Finnish

426 Cardiovascular Research Center - Tampere, Faculty of Medicine and Health Technology,

427 Tampere University, Tampere 33014, Finland, ${ }^{232}$ University of Queensland Diamantina

428 Institute, Translational Research Institute, Kent St, Woolloongabba, Brisbane, QLD, 4102,

429 Australia, ${ }^{23}$ Department of Medicine, Bornholms Hospital, Rønne, Denmark, ${ }^{234}$ School of

430 Public Health, University of Alabama at Birmingham, AL, USA, ${ }^{23}$ Cardiology, Division

431 Heart \& Lungs, University Medical Center Utrecht, Utrecht University, Utrecht, the

432 Netherlands, ${ }^{236}$ Department of Population and Quantitative Health Sciences, Case Western

433 Reserve University, Cleveland, OH, 44106, USA, ${ }^{23}$ Department of Genetics, Stanford

434 University School of Medicine Stanford, CA 94305, USA, ${ }^{238}$ Department of Epidemiology -

435 Erasmus MC - University Medical Center Rotterdam, Rotterdam, the Netherlands, ${ }^{239}$ Ohio

436 State University, Division of Endricinology, Columbus OH 43210, USA, ${ }^{240}$ University of

437 Washington, Department of Epidemiology, Seattle WA 98195, USA, ${ }^{24}$ George Washington

438 University, School of Medicine and Health Sciences, Washington DC 20037, USA,

$439{ }^{242}$ Department of Epidemiology, School of Public Health, Peking University Health Science

440 Center, Beijing, China, ${ }^{24}$ Institute for Laboratory Medicine, University Hospital Leipzig,

441 Paul-List-Strasse 13/15, 04103 Leipzig, Germany, ${ }^{24}$ Laboratory of Epidemiology and

442 Population Sciences, National Institute on Aging, NIH, Baltimore,MD, 20892-9205, USA,

$443{ }^{24}$ Shanghai Institute of Nutrition and Health, University of Chinese Academy of Sciences,

444 Chinese Academy of Sciences, Shanghai, China, ${ }^{24}$ Centre for Population Health Research,

445 University of Turku and Turku University Hospital, Finland, ${ }^{24}$ Research Centre of Applied

446 and Preventive Cardiovascular Medicine, University of Turku, Finland, ${ }^{248}$ Department of

447 Clinical Physiology and Nuclear Medicine, Turku University Hospital, Turku, Finland,

$448{ }^{24}$ Department of Environmental and Preventive Medicine, Jichi Medical University School of 
449 Medicine, Shimotsuke, 329-0498, Japan, ${ }^{25}$ Centre for Population Health Sciences, Usher

450 Institute, University of Edinburgh, Teviot Place, Edinburgh, EH8 9AG, Scotland,

$451 \quad{ }^{25}$ Department of Functional Pathology, Shimane University School of Medicine, Izumo,

452 6938501, Japan, ${ }^{252}$ Department of Pediatrics and Adolescent Medicine, Turku University

453 Hospital and University of Turku, Turku, Finland, ${ }^{253}$ Department of Physiology, University of

454 Turku, Turku, Finland, ${ }^{25}$ Faculty of Medicine, University of Split, Šoltanska 2, HR-21000,

455 Split, Croatia, ${ }^{25}$ Medical Department III - Endocrinology, Nephrology, Rheumatology,

456 University of Leipzig Medical Center, Liebigstr. 21, 04103 Leipzig, Germany, ${ }^{25}$ Department

457 of Nutrition-Dietetics, Harokopio University, Eleftheriou Venizelou, Athens, 17676, Greece,

$458{ }^{25}$ Department of Clinical Gene Therapy, Osaka University Graduate School of Medicine,

459 Suita, 5650871, Japan, ${ }^{258}$ Department of Geriatric and General Medicine, Osaka University

460 Graduate School of Medicine, Suita, 5650871, Japan, ${ }^{259}$ Department of Vascular Surgery,

461 Division of Surgical Specialties, University Medical Center Utrecht, Utrecht University,

462 Utrecht, the Netherlands, ${ }^{260}$ Corneal Dystrophy Research Institute, Department of

463 Ophthalmology, Yonsei University College of Medicine, Seoul 03722, Korea, ${ }^{261}$ Dept of

464 Radiology and Nuclear Medicine, Erasmus MC - University Medical Center Rotterdam,

465 Rotterdam, the Netherlands, ${ }^{262}$ Julius Centre for Health Sciences and Primary Care, University

466 Medical Centre Utrecht, 3584CG, the Netherlands, ${ }^{263}$ Second Department of Cardiology,

467 Medical School, National and Kapodistrian University of Athens, Attikon University

468 Hospital, Athens, Greece, ${ }^{204}$ Center for Vision Research, Department of Ophthalmology and

469 The Westmead Institute, University of Sydney, Hawkesbury Rd, Sydney, New South Wales,

470 2145, Australia, ${ }^{265}$ Menzies Institute for Medical Research, School of Medicine, University of

471 Tasmania, Liverpool St, Hobart, Tasmania, 7000, Australia, ${ }^{26}$ Centre for Eye Research

472 Australia, University of Melbourne, Melbourne, Victoria, 3002, Australia, ${ }^{26}$ Department of

473 Clinical Physiology, Tampere University Hospital, Tampere 33521, Finland, ${ }^{268}$ Department of 
474 Clinical Physiology, Finnish Cardiovascular Research Center - Tampere, Faculty of Medicine

475 and Health Technology, Tampere University, Tampere 33014, Finland, ${ }^{20}$ Centre Nutrition,

476 santé et société (NUTRISS), Institute of Nutrition and Functional Foods (INAF), Québec,

477 Canada, ${ }^{27}$ Pennington Biomedical Research Center, Baton Rouge, LA 70808, USA, ${ }^{27}$ Medical

478 Department III - Endocrinology, Nephrology, Rheumatology, University of Leipzig Medical

479 Center, Liebigstr. 18, 04103 Leipzig, Germany, ${ }^{272}$ Discipline of Internal Medicine, Medical

480 School, The University of Western Australia, Perth, WA, Australia, ${ }^{273}$ Institute of

481 Epidemiology, Kiel University, Kiel, Germany, ${ }^{27}$ Institute of Clinical Molecular Biology,

482 Kiel University, Kiel, Germany, ${ }^{27}$ Sahlgrenska University Hospital, Department of Drug

483 Treatment, Gothenburg, Sweden, ${ }^{276}$ Geriatric Medicine, Institute of Medicine, Sahlgrenska

484 Academy, University of Gothenburg, Gothenburg, Sweden, ${ }^{27}$ Department of Internal

485 Medicine, EwhaWomans University School of Medicine, Seoul, Korea, ${ }^{278}$ Division of Cancer

486 Control and Population Sciences, UPMC Hillman Cancer Center, University of Pittsburgh,

487 Pittsburgh, PA 15232, USA, ${ }^{279}$ Department of Epidemiology, Graduate School of Public

488 Health, University of Pittsburgh, Pittsburgh, PA 15232, USA, ${ }^{28}$ Healthy Longevity

489 Translational Research Programme, Yong Loo Lin School of Medicine, National University

490 of Singapore, Singapore 117545, Singapore, ${ }^{281}$ Singapore Institute for Clinical Sciences,

491 Agency for Science Technology and Research (A*STAR), Singapore 117609, Singapore,

$492 \quad{ }^{282}$ Department of Endocrinology and Metabolism, Kyung Hee University School of Medicine,

493 Seoul 02447, Korea, ${ }^{283}$ Institute for Community Medicine, University Medicine Greifswald,

494 Germany, ${ }^{284}$ Laboratory of Epidemiology and Population Science National Institute on Aging

495 Intramural Research Program, NIH 251 Bayview Blvd, NIH Biomedical Research Center,

496 Baltimore, MD 21224, USA, ${ }^{285}$ Algebra University College, Ilica 242, Zagreb, Croatia,

$497 \quad{ }^{28}$ Paavo Nurmi Centre, Sports and Exercise Medicine Unit, Department of Physical Activity

498 and Health, University of Turku, Turku, Finland, ${ }^{28}$ Interdisciplinary Center Psychopathology 
499 and Emotion Regulation (ICPE), University of Groningen, University Medical Center

500 Groningen, Groningen, 9700 RB, the Netherlands, ${ }^{28}$ Institute of Molecular Genetics, National

501 Research Council of Italy, Pavia, Italy, ${ }^{28}$ Gottfried Schatz Research Center for Cell Signaling,

502 Metabolism and Aging, Medical University of Graz, Graz, Austria, ${ }^{20}$ Local Health Unit

503 Toscana Centro, Firenze, Italy, ${ }^{29}$ Institute of Nutritional Science, University of Potsdam,

504 Nuthetal, Germany, ${ }^{22}$ Department of Medicine, Surgery and Health Sciences, University of

505 Trieste, Strada di Fiume 447, 34149, Trieste, Italy, ${ }^{293}$ Dept of Nephrology, Diabetology,

506 Rheumatology; Traunstein Hospital, Traunstein, Germany, ${ }^{24} \mathrm{KfH}$ Kidney Center Traunstein,

507 Traunstein, Germany, ${ }^{295}$ Center for Translational and Systems Neuroimmunology, Department

508 of Neurology, Columbia University Medical Center, New York, NY, USA, ${ }^{20}$ Program in

509 Medical and Population Genetics, Broad Institute, Cambridge, MA, USA, ${ }^{29}$ Medical School,

510 National and Kapodistrian University Athens, 75 M. Assias Street, 11527 Athens, Greece,

$511{ }^{298}$ Dromokaiteio Psychiatric Hospital, 12461 Athens, Greece, ${ }^{29}$ Clinical Pharmacology,

512 William Harvey Research Institute, Queen Mary University of London, London, EC1M

513 6BQ,UK, ${ }^{300}$ Department of Ophthalmology, Medical Faculty Mannheim, Heidelberg

514 University, Kutzerufer 1, Mannheim, 68167, Germany, ${ }^{30}$ Institute of Molecular and Clinical

515 Ophthalmology Basel, Switzerland, ${ }^{30}$ Privatpraxis Prof Jonas und Dr Panda-Jonas,

516 Heidelberg, Germany, ${ }^{30}$ Department of Human Genetics, David Geffen School of Medicine at

517 UCLA, University of California, Los Angeles, CA, USA, ${ }^{30}$ Unidad de Biología Molecular y

518 Medicina Genómica, Instituto Nacional de Ciencias Médicas y Nutrición Salvador Zubirán,

519 Mexico 14080, Mexico, ${ }^{30}$ Instituto de Investigaciones Biomédicas, UNAM, Mexico,

$520{ }^{306}$ Departamento de Endocrinología y Metabolismo, Instituto Nacional de Ciencias Médicas y

521 Nutrición Salvador Zubirán, Mexico 14080, Mexico, ${ }^{30}$ Department of Nutrition, Gillings

522 School of Global Public Health, University of North Carolina, Chapel Hill, North Carolina,

52327599 USA, ${ }^{300}$ Carolina Population Center, University of North Carolina, Chapel Hill, North 
524 Carolina, 27516 USA, ${ }^{30}$ USC-Office of Population Studies Foundation, University of San

525 Carlos, Cebu City, 6000, Philippines, ${ }^{310}$ Department of Anthropology, Sociology, and History,

526 University of San Carlos, Cebu City, 6000 Philippines, ${ }^{31}$ Department of Medicine, Faculty of

527 Medicine, University of Kelaniya, Ragama, 11010, Sri Lanka, ${ }^{32}$ Department of Public Health,

528 Faculty of Medicine, University of Kelaniya, Ragama, 11010, Sri Lanka, ${ }^{313}$ Children's

529 Hospital Oakland Research Institute, Oakland, CA 94609 USA, ${ }^{314}$ Institute of Biomedical

530 Sciences, Academia Sinica, Taiwan, ${ }^{315}$ Systems Genomics Laboratory, School of

531 Biotechnology, Jawaharlal Nehru University, New Delhi - 110067, India, ${ }^{316}$ Department of

532 Medicine, University of Mississippi Medical Center, Jackson, MS, 39216, USA,

$533{ }^{317}$ Department of Physiology and Biophysics, University of Mississippi Medical Center,

534 Jackson, MS, 39216, USA, ${ }^{318}$ Department of Medical Sciences, Uppsala University, Sweden,

$535{ }^{319}$ Department of Paediatrics, Yong Loo Lin School of Medicine, National University of

536 Singapore; and Khoo Teck Puat - National University Children's Medical Institute, National

537 University Health System, Singapore, ${ }^{320}$ Department of Medicine, University of North

538 Carolina, Chapel Hill, NC, USA, ${ }^{32}$ Department of Epidemiology, Gillings School of Global

539 Public Health, University of North Carolina, Chapel Hill, North Carolina, USA, ${ }^{32}$ Injury

540 Prevention Research Center, University of North Carolina, Chapel Hill, North Carolina,

541 USA, ${ }^{32}$ Division of Physical Therapy, University of North Carolina, Chapel Hill, North

542 Carolina, USA, ${ }^{32}$ Department of Psychiatry, Amsterdam UMC, Vrije Universiteit

543 Amsterdam, the Netherlands, ${ }^{325}$ Amsterdam Public Health research institute, VU medical

544 center Amsterdam, the Netherlands, ${ }^{326}$ Department of Biochemistry, College of Medicine,

545 Ewha Womans University, Seoul 07804, Korea, ${ }^{32}$ Faculty of Health and Medicine, University

546 of Newcastle, Australia, ${ }^{328}$ Washington University School of Medicine, Division of

547 Biostatistics, MO, USA, ${ }^{32}$ University of Kentucky, College of Public Health, KY, USA,

$548{ }^{33}$ Institute of Cellular Medicine (Diabetes), The Medical School, Newcastle University, 
549 Framlington Place, Newcastle upon Tyne, NE2 4HH, UK, ${ }^{33}$ University of Helsinki and

550 Department of Medicine, Helsinki University Hospital, P.O.Box 340, Haartmaninkatu 4,

551 Helsinki, FI-00029, Finland, ${ }^{32}$ Minerva Foundation Institute for Medical Research,

552 Biomedicum 2U, Tukholmankatu 8, Helsinki, FI-00290, Finland, ${ }^{33}$ JSS Academy of Higher

553 Education and Research, Mysuru, India, ${ }^{33}$ Programs in Metabolism and Medical and

554 Population Genetics, Broad Institute of MIT and Harvard, Cambridge, MA, USA, ${ }^{335}$ Diabetes

555 Unit and Center for Genomic Medicine, Massachusetts General Hospital, Boston, MA,

556 USA10, ${ }^{336}$ Harvard Medical School, Boston, Massachusetts, USA, ${ }^{337}$ Unidad de Biología

557 Molecular y Medicina Genómica, Instituto de Investigaciones Bimédicas UNAM/ Instituto

558 Nacional de Ciencias Médicas y Nutrición Salvador Zubirán, Mexico City, Mexico,

559 ${ }^{33}$ Dirección de Nutrición and Unidad de Estudios de Enfermedades Metabólicas, Instituto

560 Nacional de Ciencias Médicas y Nutrición Salvador Zubirán, Mexico City, Mexico,

$561 \quad{ }^{33}$ InstitutoNacional de Salud Publica y Centro de Estudios en Diabetes, Mexico, ${ }^{340}$ Instituto

562 Nacional de Medicina Genómica, Mexico, ${ }^{34}$ Human Genetics Center, School of Public

563 Health, University of Texas Health Science Center at Houston, Houston TX 77030, USA,

$564 \quad{ }^{342}$ Yong Loo Lin School of Medicine, National University of Singapore and National

565 University Health System, 119228, Singapore, ${ }^{343}$ MRC Unit for Lifelong Health and Ageing at

566 UCL, 1-19 Torrington Place, London, WC1E 7HB, United Kingdom, ${ }^{34}$ Kurume University

567 School of Medicine, Kurume, 830-0011, Japan, ${ }^{345}$ Genetics, Merck Sharp \& Dohme Corp.,

568 Kenilworth, NJ, 07033, USA, ${ }^{346}$ Oxford Centre for Diabetes, Endocrinology \& Metabolism,

569 University of Oxford, UK, ${ }^{34}$ Population Health and Genomics, University of Dundee,

570 Ninwells Hospital and Medical School, Dundee, DD1 9SY, UK, ${ }^{348}$ Intramural Research

571 Program, National Institute on Aging, 3001 S. Hanover St., Baltimore, MD 21225, ${ }^{34}$ Beijing

572 Institute of Ophthalmology, Beijing Tongren Eye Center, Beijing Tongren Hospital, Capital

573 Medical University, Beijing Ophthalmology and Visual Sciences Key Laboratory, 100730 
574 Beijing, China, ${ }^{350}$ The Eye Hospital, School of Ophthalmology \& Optometry, Wenzhou

575 Medical University, Wenzhou, Zhejiang 325027, China, ${ }^{35}$ Synlab Academy, SYNLAB

576 Holding Deutschland GmbH, Mannheim and Augsburg, Germany, ${ }^{352}$ Clinical Institute of

577 Medical and Chemical Laboratory Diagnostics, Medical University of Graz, Austria,

$578{ }^{353}$ Faculty of Medicine, University of Iceland, 101 Reykjavik, Iceland, ${ }^{354}$ Leiden University

579 Medical Center, Department of Cell and Chemical Biology, Leiden, 2333ZA, The

580 Netherlands, ${ }^{355}$ Leiden University Medical Center, Department of Biomedical Data Sciences,

581 Section Molecular Epidemiology, Leiden, 2333ZA, The Netherlands, ${ }^{356}$ Amsterdam UMC,

582 Department of Epidemiology and Data Science, Amsterdam Public Health Research Institute,

583 Amsterdam, 1081HV, the Netherlands., ${ }^{357}$ Amsterdam UMC, Department of General Practice

584 and Elderly Care, Amsterdam Public Health Research Institute, Amsterdam, 1081HV, The

585 Netherlands, ${ }^{358}$ Department of Genetics, University of Pennsylvania, Philadelphia, PA, 19104,

586 USA, ${ }^{35}$ Department of Surgery, University of Pennsylvania, Philadelphia, PA, 19104, USA,

$587{ }^{360}$ Corporal Michael Crescenz VA Medical Center, Philadelphia, Pennsylvania, PA, 19104,

588 USA, ${ }^{36 ! I n s t i t u t e ~ o f ~ S o c i a l ~ a n d ~ E c o n o m i c ~ R e s e a r c h, ~ U n i v e r s i t y ~ o f ~ E s s e x, ~ W i v e n h o e ~ P a r k, ~ C O 4 ~}$

589 3SQ, United Kingdom, ${ }^{36}$ Department of Cardiology, University of Groningen, University

590 Medical Center Groningen, 9700RB Groningen, The Netherlands, ${ }^{36}$ Department of

591 Environmental Medicine and Public Health, Icahn School of Medicine at Mount Sinai, New

592 York, NY, USA, ${ }^{364}$ Unidad de Investigacion Medica en Bioquimica, Hospital de

593 Especialidades, Centro Medico Nacional Siglo XXI, Instituto Mexicano del Seguro Social,

594 Mexico City, Mexico., ${ }^{365}$ Department of Epidemiology, University of Washington, Seattle,

595 WA, USA, ${ }^{36}$ Department of Health Services, University of Washington, Seattle, WA, USA,

$596{ }^{36}$ Institute of Regional Health Research, University of Southern Denmark, Odense, Denmark,

$597{ }^{368} 16 C e n t e r$ for Research on Genomics and Global Health, National Human Genome Research

598 Institute, National Institutes of Health, 12 South Drive, Room 4047, Bethesda, MD, 20892, 
599 USA,, ${ }^{36}$ Danish Aging Research Center, University of Southern Denmark; Odense C,

600 Denmark, ${ }^{370}$ Public Health, Faculty of Medicine, University of Helsinki, Finland, ${ }^{37}$ Broad

601 Institute of MIT and Harvard, Cambridge, MA, ${ }^{372}$ Center for Applied Genomics, Children's

602 Hospital of Philadelphia, Philadelphia, PA, 19104 USA, ${ }^{373}$ Department of Pediatrics, The

603 University of Pennsylvania Perelman School of Medicine, Philadelphia, PA, 19104 USA,

$604{ }^{37}$ Division of Human Genetics, Children's Hospital of Philadelphia, Philadelphia, PA, 19104

605 USA, ${ }^{37}$ Department of Genetics, University of Pennsylvania, Philadelphia, PA, 19104 USA,

$606{ }^{37}$ Radboud university medical center, Radboud Institute for Health Sciences, Nijmegen, The

607 Netherlands, ${ }^{37}$ School of Medicine, Southern University of Science and Technology,

608 Shenzhen, China, ${ }^{378}$ Institute for Cardiogenetics, University of Lübeck, DZHK (German

609 Research Centre for Cardiovascular Research), partner site Hamburg/Lübeck/Kiel, and

610 University Heart Center Lübeck, Lübeck, Germany, ${ }^{37}$ Netherlands Heart Institute, Utrecht,

611 the Netherlands, ${ }^{380}$ Division of Cardiology, Department of Medicine, Massachusetts General

612 Hospital, Boston, Massachusetts, USA, ${ }^{38}$ Program of Medical and Population Genetics, Broad

613 Institute, Cambridge, Massachusetts, USA, ${ }^{382}$ Center for Genomic Medicine, Massachusetts

614 General Hospital, Boston, Massachusetts, USA, ${ }^{38}$ Department of Medicine, Harvard Medical

615 School, Boston, Massachusetts, USA, ${ }^{384}$ Northern Finland Birth Cohorts, Infrastructure for

616 population studies, Faculty of Medicine, University of Oulu, Oulu, Finland, ${ }^{385}$ Center for Life

617 Course Health Research, Faculty of Medicine, University of Oulu, Oulu, Finland, ${ }^{386}$ Biocenter

618 of Oulu, University of Oulu, Oulu, Finland, ${ }^{38}$ Institute for Genetic and Biomedical Research,

619 Italian National Council of Research (IRGB CNR), Cagliari, Italy, ${ }^{388}$ University of Sassari,

620 Sassari, Italy, ${ }^{38}$ Department of Clinical Epidemiology, Leiden University Medical Center,

621 Leiden, the Netherlands, ${ }^{30}$ Department of Public Health and Primary Care, Leiden University

622 Medical Center, Leiden, the Netherlands, ${ }^{39}$ Department of Internal Medicine, Division of

623 Endocrinology, Leiden University Medical Center, Leiden, the Netherlands, ${ }^{32}$ Einthoven 
624 Laboratory for Experimental Vascular Medicine, Leiden University Medical Center, Leiden,

625 the Netherlands, ${ }^{393}$ Department of Human Genetics, Leiden University Medical Center,

626 Leiden, the Netherlands, ${ }^{34}$ Population Health Research Institute, St George's, University of

627 London, London SW17 ORE, UK, ${ }^{395}$ National Heart and Lung Institute, Imperial College

628 London, London, W2 1PG, UK, ${ }^{396}$ School of Public Health, Imperial College London,

629 London, W12 7RH, UK, ${ }^{39}$ Taichung Veterans General Hospital, Taichung, Taiwan; No. 1650,

630 Sec. 4, Taiwan Boulevard, Xitun District Taichung City 40705, Taiwan, ${ }^{398}$ Division of

631 Endocrinology and Metabolism, Department of Medicine, Taipei Veterans General Hospital,

632 Taipei, Taiwan; No. 201, Sec. 2, Shipai Road, Beitou District, Taipei City, 112201, Taiwan,

$633{ }^{39}$ OCDEM, University of Oxford, Churchill Hospital, Oxford OX3 7LE, UK, ${ }^{400}$ NIHR Oxford

634 Biomedical Research Centre, Churchill Hospital, Oxford, UK, ${ }^{40}$ Ocular Epidemiology,

635 Singapore Eye Research Institute, Singapore National Eye Centre, 168751, Singapore,

$636{ }^{402}$ Ophthalmology \& Visual Sciences Academic Clinical Program (Eye ACP), Duke-NUS

637 Medical School, 169857, Singapore, ${ }^{403}$ Data Science, Singapore Eye Research Institute,

638 Singapore National Eye Centre, 168751, Singapore, ${ }^{404}$ DZHK (German Centre for

639 Cardiovascular Research), Munich Heart Alliance partner site, Munich, Germany, ${ }^{40}$ German

640 Center for Diabetes Research (DZD), Neuherberg, Germany, ${ }^{406}$ University of Exeter Medical

641 School, University of Exeter, Exeter, EX2 5DW, UK, ${ }^{407}$ Department of Medical Epidemiology

642 and Biostatistics, Karolinska Institutet, Stockholm, Sweden, ${ }^{408}$ Amsterdam Public Health

643 research institute, Amsterdam UMC, the Netherlands, ${ }^{40}$ Framingham Heart Study, National

644 Heart, Lung, and Blood Institute, US National Institutes of Health, Bethesda, MD, USA.,

$645{ }^{40}$ Department of Genetics, School of Medicine, Mashhad University of Medical Sciences,

646 Mashhad, Iran, ${ }^{41}$ Department of Genetics, Shanghai-MOST Key Laboratory of Health and

647 Disease Genomics, Chinese National Human Genome Center at Shanghai, Shanghai, 201203

648 China, ${ }^{412}$ Technical University of Munich (TUM) and Klinikum Rechts der Isar, TUM School 
649 of Medicine, Munich, Germany, ${ }^{413}$ Department of Public Health, University of Helsinki,

650 Helsinki, Finland, ${ }^{44}$ Diabetes Research Group, King Abdulaziz University, Jeddah, Saudi

651 Arabia, ${ }^{415}$ Institute of Clinical Medicine, Internal Medicine, University of Eastern Finland and

652 Kuopio University Hospital, Finland, ${ }^{416}$ Stanford Cardiovascular Institute, Stanford

653 University, Stanford, CA 94305, USA, ${ }^{417}$ Stanford Diabetes Research Center, Stanford

654 University, Stanford, CA 94305, USA, ${ }^{48}$ Department of Medical Sciences, Molecular

655 Epidemiology and Science for Life Laboratory, Uppsala University, Uppsala, Sweden.,

$656{ }^{410}$ Regeneron Pharmaceuticals, Tarrytown, NY, USA, ${ }^{420}$ Lee Kong Chian School of Medicine,

657 Nanyang Technological University, Singapore 308232, Singapore, ${ }^{42}$ Imperial College

658 Healthcare NHS Trust, Imperial College London, London W12 0HS, UK, ${ }^{422}$ MRC-PHE

659 Centre for Environment and Health, Imperial College London, London W2 1PG, UK,

$660{ }^{423}$ National Heart and Lung Institute, Imperial College London, London W12 0NN, UK,

$661{ }^{424}$ School of Electrical \& Information Engineering, University of the Witwatersrand, South

662 Africa, ${ }^{42}$ Department of Epidemiology, University of North Carolina, Chapel Hill, NC, USA,

$663{ }^{426}$ Institute for Minority Health Research, University of Illinois College of Medicine, Chicago,

664 Illinois, USA, ${ }^{42}$ Department of Biostatistics, Harvard T.H. Chan School of Public Health, 677

665 Huntington Avenue, Boston, MA, 02115, USA, ${ }^{428}$ QIMR Berghofer Medical Research

666 Institute, 300 Herston Road, Brisbane, Queensland 4006, Australia, ${ }^{42}$ Center for Non-

667 Communicable Diseases, Karachi, Sindh, Pakistan \& Faisalabad Institute of Cardiology,

668 Faislabad, Pakistan, ${ }^{430}$ Department of Medicine, Columbia University Irving Medical Center,

669 New York, NY, USA, ${ }^{43}$ Department of Cardiology, Columbia University Irving Medical

670 Center, New York, NY, USA, ${ }^{432}$ Big Data Instutute, University of Oxford, Oxford OX3 7LF,

671 UK, ${ }^{433}$ National Institute for Health Research Oxford Biomedical Research Centre, Oxford

672 University Hospitals, Oxford, UK, ${ }^{43}$ Aberdeen Centre for Health Data Science, 1:042

673 Polwarth Building School of Medicine, Medical Science and Nutrition University of 
674 Aberdeen, Foresterhill, Aberdeen, AB25 2ZD, UK, ${ }^{43}$ Division of Population Health and

675 Genomics, Ninewells Hospital and Medical School, University of Dundee, Dundee DD1

676 9SY, United Kingdom, ${ }^{436}$ Biomedical and Translational Informatics, Geisinger Health,

677 Danville, PA 17822, USA, ${ }^{43}$ Harvard Medical School, Boston, MA 02115, USA, ${ }^{438}$ School of

678 Basic and Medical Biosciences, Faculty of Life Sciences and Medicine, King's College

679 London, London, UK, ${ }^{439}$ Department of Biomedical Informatics, Vanderbilt University

680 Medical Center, Nashville, TN, USA, ${ }^{40}$ Departments of Medicine (Medical Genetics) and

681 Genome Sciences, University of Washington, USA, ${ }^{44}$ Center for Autoimmune Genomics and

682 Etiology, Cincinnati Children's Hospital Medical Center (CCHMC), Cincinnati, OH, USA.,

$683{ }^{42}$ Division of Endocrinology, Metabolism, and Molecular Medicine, Department of Medicine,

684 Northwestern University, Feinberg School of Medicine, Chicago, IL 60611, USA,

$685{ }^{43}$ Department of Anthropology, Northwestern University, Evanston, IL 60208, USA, ${ }^{44}$ Center

686 for Genetic Medicine, Northwestern University, Feinberg School of Medicine, Chicago, IL

687 60611, USA, ${ }^{45}$ HUNT Research Centre, Department of Public Health and Nursing, NTNU,

688 Norwegian University of Science and Technology, Levanger, 7600 Norway, ${ }^{46}$ Department of

689 Medicine, Levanger Hospital, Nord-Trøndelag Hospital Trust, Levanger, 7600 Norway,

$690{ }^{44}$ Department of Endocrinology, St. Olavs Hospital, Trondheim University Hospital,

691 Trondheim, Norway, ${ }^{44}$ RIKEN Center for Integrative Medical Sciences, Yokohama, Japan,

$692{ }^{44}$ Laboratory of Complex Trait Genomics, Department of Computational Biology and

693 Medical Sciences, Graduate School of Frontier Sciences, The University of Tokyo, Tokyo,

694 Japan, ${ }^{450}$ Laboratory of Statistical Immunology, WPI Immunology Frontier Research Center,

695 Osaka University, Osaka, Japan, ${ }^{45}$ Integrated Frontier Research for Medical Science Division,

696 Institute for Open and Transdisciplinary Research Initiatives, Osaka University, Osaka,

697 Japan, ${ }^{452}$ Division of Molecular Pathology, Institute of Medical Science, The University of

698 Tokyo, Tokyo, Japan, ${ }^{453}$ Division of Genome Research, Center for Genome Science, National 
699 Institute of Health, Chungcheongbuk-do, South Korea, ${ }^{454}$ Faculty of Medicine, University of

700 Iceland, Sæmundargötu 2, Reykjavik, 102, Iceland, ${ }^{455}$ VA Boston Healthcare System, Boston,

701 MA, USA, ${ }^{456}$ VA Informatics and Computing Infrastructure, VA Salt Lake City Health Care

702 System, Salt Lake City, UT, USA, ${ }^{45}$ University of Massachusetts, Boston, MA, USA,

$703{ }^{458}$ Department of Medicine, University of Pennsylvania Perelman School of Medicine,

704 Philadelphia, PA, USA, ${ }^{45}$ Cardiovascular Institute, Stanford University School of Medicine,

705 Stanford, California, USA, ${ }^{460}$ Corporal Michael J. Crescenz VA Medical Center, Philadelphia,

706 PA, USA, ${ }^{461}$ Department of Medicine, Brigham Women's Hospital, Boston, MA, USA,

$707{ }^{462}$ Division of Cardiology, Emory University School of Medicine, Atlanta, GA, USA,

708 ${ }^{463}$ Endocrinology, Boston Childrens Hospital, Boston 02115 MA, USA, ${ }^{464}$ Departments of

709 Pediatrics and Genetics, Harvard Medical School, Boston, MA, USA, ${ }^{46}$ Center for Genomic

710 Medicine, Department of Medicine, Massachusetts General Hospital, Boston, Massachusetts,

711 USA, ${ }^{46}$ Cardiology Division, Massachusetts General Hospital, Harvard Medical School,

712 Boston, MA, USA, ${ }^{46}$ Department of Medicine, Massachusetts General Hospital, Harvard

713 Medical School, Boston, MA, USA, ${ }^{468}$ Cardiovascular Research Center and Center for

714 Genomic Medicine, Massachusetts General Hospital, Boston, MA, USA, ${ }^{46}$ Centre for

715 Genetics and Genomics Versus Arthritis, Centre for Musculoskeletal Research, Division of

716 Musculoskeletal and Dermatological Sciences, The University of Manchester, Manchester,

717 UK, ${ }^{470}$ Princess Al-Jawhara Al-Brahim Centre of Excellence in Research of Hereditary

718 Disorders (PACER-HD), King Abdulaziz University, Jeddah, Saudi Arabia, ${ }^{471}$ Department of

719 Biostatistics, Boston University School of Public Health, Boston, MA, USA, ${ }^{42}$ Department of

720 Human Genetics, University of Michigan, Ann Arbor, MI, 48109, USA, ${ }^{473}$ Department of

721 Statistics, The Pennsylvania State University, University Park, PA, USA, ${ }^{47}$ Huck Institutes of

722 the Life Sciences, The Pennsylvania State University, University Park, PA, USA, ${ }^{475}$ VA Palo 
723 Alto Health Care System, Palo Alto, CA, USA, ${ }^{47}$ Department of Statistics, Stanford

724 University, Stanford, CA, USA

725 Corresponding Authors:

726 Xiang Zhu, PhD

727 Department of Statistics

728 Huck Institutes of the Life Sciences

729 The Pennsylvania State University

730 University Park, PA 16802

731 xiangzhu@psu.edu

732

733 Christopher D Brown, PhD

734 Department of Genetics

735 Perelman School of Medicine

736 University of Pennsylvania

737 Philadelphia PA 19104

738 chrbro@upenn.edu 
741 A major challenge of genome-wide association studies (GWAS) is to translate phenotypic

742 associations into biological insights. Here, we integrate a large GWAS on blood lipids

743 involving 1.6 million individuals from five ancestries with a wide array of functional

744 genomic datasets to discover regulatory mechanisms underlying lipid associations. We first

745 prioritize lipid-associated genes with expression quantitative trait locus (eQTL)

746 colocalizations, and then add chromatin interaction data to narrow the search for functional

747 genes. Polygenic enrichment analysis across 697 annotations from a host of tissues and cell

748 types confirms the central role of the liver in lipid levels, and highlights the selective

749 enrichment of adipose-specific chromatin marks in high-density lipoprotein cholesterol and

750 triglycerides. Overlapping transcription factor (TF) binding sites with lipid-associated loci

751 identifies TFs relevant in lipid biology. In addition, we present an integrative framework to

752 prioritize causal variants at GWAS loci, producing a comprehensive list of candidate causal

753 genes and variants with multiple layers of functional evidence. Two prioritized genes,

$754 C R E B R F$ and $R R B P 1$, show convergent evidence across functional datasets supporting their 755 roles in lipid biology.

756

757 
758

759

760

761

762

763

764

765

766

767

768

769

770

771

772

773

774

775

776

777

778

779

780

781

782

\section{Introduction}

Most GWAS findings have not directly led to mechanistic interpretations, largely because $90 \%$ of GWAS associations map to non-coding sequences ${ }^{1,2}$. Mechanistic interpretations in GWAS have proven challenging because the strongest signals identified in GWAS typically contain many variants in strong linkage disequilibrium (LD) ${ }^{3}$ and functional mechanisms including genes of action are often not clear from GWAS data alone ${ }^{4,5}$.

Linking trait-associated variants to genome function has emerged as a promising model for mechanistic interpretation of non-coding findings in GWAS. This 'variant-to-function' model is premised on recent observations that non-coding variants often affect a trait of interest through the regulation of genes and processes in trait-relevant cell types or tissues ${ }^{2,6}$. Implementing this functional model in GWAS has become more feasible as large-scale functional genomic resources, such as epigenomic ${ }^{7}$ and transcriptomic ${ }^{8}$ catalogues, have been systematically generated across a wide range of human cell types and tissues. The integration of functional genomics with GWAS has identified regulatory mechanisms in variants associated with some flagship disorders such as obesity ${ }^{9}$ and schizophrenia ${ }^{10}$, yielding important functional insights into the genetic architecture of human complex traits.

The history of the human genetics of lipids mirrors the successes and challenges of GWAS. Increasing sample size and genetic diversity has significantly boosted the power of discovery: the first lipid GWAS in 2008 with 8,816 European-descent individuals identified 29 lipidassociated loci ${ }^{11}$; the latest study of 1.6 million individuals across five ancestries ${ }^{12}$ found 941. Despite the dramatic increase in the number of associations, our biological understanding of many of these genetic discoveries remains limited. The causal gene has 
783 been confidently assigned at only a small fraction of these loci ${ }^{2}$, and the regulatory

784 mechanism connecting variant to phenotype has been conclusively characterized only for a

785 handful of genes ${ }^{5}$. Furthermore, systematic mapping of lipid-associated variants to their

786 biological functions has been missing in the literature at the time of this study.

788 Here we conduct a genome-scale integrative analysis on the largest GWAS to-date of five

789 lipid phenotypes (LDL, or low density lipoprotein; HDL, or high density lipoprotein; TC, or

790 total cholesterol; nonHDL, or non-high density lipoprotein; and TG, or triglycerides)

791 involving 1.65 million individuals from five ancestries ${ }^{12}$. Combining the lipid GWAS with a

792 wide array of functional genomic resources in diverse human tissues and cell types, we

793 identify regulatory mechanisms of noncoding genetic variation in lipids with a full suite of

794 computational approaches. Further, we develop a generalizable framework to understand how

795 tissue-specific gene regulation can explain GWAS findings, and demonstrate its real-world

796 value on lipid-associated loci.

797

798 Material and Methods

799 GWAS

800

801 We performed GWAS for five blood lipid traits (LDL, HDL, TC, TG, and nonHDL) in 1.65

802 million individuals from five ancestry groups ${ }^{12}$ (African and African-admixed, East Asian,

803 European, Hispanic, South Asian) at 91 million variants imputed primarily from the

804 Haplotype Reference Consortium ${ }^{13}$ or 1000 Genomes Phase $3^{14}$. The individual GWAS and

805 meta-analyses were performed using the hg19 version of the human reference genome. We

806 used MR-MEGA ${ }^{15}$ for meta-analysis across cohorts. 
808 We defined 'sentinel variants' as lead variants representing independent trait-associated loci in

809 the genome. These windows are the greater of $500 \mathrm{~kb}$ or $0.25 \mathrm{cM}$ around the sentinel variant;

810 genetic distances were defined using reference maps from HapMap $3^{16}$. We performed a

811 second round of conditional analysis, conditioning on the sentinel variants to identify and

812 remove any significant windows that are shadow signals (or dependent on) of a neighboring

813 locus to enforce independence of associated loci.

814

815 Colocalization with eQTLs

816

817 We performed statistical colocalization of lipid GWAS with eQTLs obtained from GTEx v8

818 across 49 tissues $^{8}$. For each of the five lipid traits, we used the same sentinel variants defined

819 in the previous section to represent approximately independent GWAS-associated windows

820 (also removing shadow signals as described before).

821

822 For each such window, we ran eQTL colocalization with GTEx v8 single-tissue cis-eQTL

823 summary statistics ${ }^{8}$. For each of 49 GTEx tissues, we first identified all genes within $1 \mathrm{Mb}$ of

824 the sentinel SNP, and then restricted analysis to those genes with significant eQTLs (i.e.,

825 'eGenes' as defined by GTEx) in that tissue (FDR < 0.05). We used the R package 'coloc' (run

826 on $\mathrm{R}$ version 3.4.3, coloc version 3.2.1) ${ }^{17}$ with default parameters to run colocalization

827 between the GWAS signal and the eQTL signal for each of these cis-eGenes, using as input

828 those SNPs in the defined window (greater than $500 \mathrm{~kb}$ or $0.25 \mathrm{cM}$ on either side of the lead

829 variant), i.e. all SNPs present in both datasets. eQTL summary statistics were in GRCh38, so

830 we first lifted over the GWAS summary statistics (in hg19) to GRCh38 using liftOver ${ }^{18}$. As

831 in previous studies ${ }^{19}$, we used a colocalization posterior probability of $(\mathrm{PP} 3+\mathrm{PP} 4)>0.8$ to

832 identify loci with enough colocalization power, and PP4/PP3 $>0.9$ to define those loci that 
833 show significant colocalization, where PP4 represents posterior probability of a single shared

834 signal, and PP3 represents posterior probability of two unique signals in the GWAS and

835 eQTL datasets.

836

Overlap with promoter Capture-C data

838

839 We used four promoter-focused Capture-C (henceforth Capture-C) datasets from three human

840 cell/tissue types to capture physical interactions between gene promoters and their regulatory

841 elements. We used three biological replicates of HepG2 liver carcinoma cells ${ }^{20}$, another

842 HepG2 dataset described in Selvarajan et al ${ }^{21}$, hepatocyte-like cells (HLC) produced by

843 differentiating three biological replicates of iPSCs (which in turn were generated from

844 peripheral blood mononuclear cells using a previously published protocol ${ }^{22}$ ), and an adipose

845 dataset obtained from Pan et al ${ }^{23}$ that was produced using primary human white adipocytes.

846

847 The detailed protocol to prepare HepG2 or HLC cells for the Capture-C experiment is

848 described in Chesi et $\mathrm{al}^{20}$. Briefly, for each dataset, 10 million cells were used for promoter

849 Capture-C library generation. Custom capture baits were designed using an Agilent

850 SureSelect library design targeting both ends of DpnII restriction fragments encompassing

851 promoters (including alternative promoters) of all human coding genes, noncoding RNA,

852 antisense RNA, snRNA, miRNA, snoRNA, and lincRNA transcripts, totaling 36,691 RNA

853 baited fragments. Each library was then sequenced on an Illumina HiSeq 4000 (HepG2) or

854 Illumina NovoSeq (HLC), generating 1.6 billion read pairs per sample (50 base pair read

855 length.) We used HiCUP v0.7. $2^{24}$ to process the raw FastQ files into loop calls and

856 CHiCAGO v1.6.0 ${ }^{24,25}$ to define significant looping interactions; we defined a CHiCAGO

857 score of 5 as significant, as specified in the default parameters. 
859 Starting with Capture-C maps processed as described above, we re-annotated the baits to 860 gene IDs from Gencode v19 ${ }^{26}$ to ensure uniformity of gene annotations with the rest of our

861 pipeline. For each bait, we identified any gene whose transcription start site (TSS) from any

862 transcript in Gencode v19 was within 175 base pair distance from the bait (to account for

863 differing bait designs for external datasets which may not directly overlap the canonical

864 TSS). We filtered all datasets to only include interactions in which the interacting end was

865 not another bait. Enrichment with colocalized genes was robust to our choice of distance

866 between bait and gene (enrichment with eQTL colocalized genes ranging from 2.94-2.96 for

867 bait distances from 0-350 base pairs).

868

869 To identify genetic variants associated with any of the five lipid traits that physically interact

870 with locations in the genome, we used the R package 'Genomic Ranges' version $1.30 .3^{27}$ to

871 find overlap between credible sets for each trait's GWAS and the previously annotated

872 promoter Capture-C data; we refer to these as Capture-C/GWAS interactions. Each credible

873 set was defined as the set of variants with a $95 \%$ posterior probability of being the causal

874 variant. For all individual variants within all GWAS-associated loci for the five lipid traits,

875 we identified which variants overlapped any interacting end of the four previously annotated

876 promoter Capture-C data.

878 Presence of gene-variant pairs in same topologically associated domains

880 To estimate the frequency of colocalized gene-sentinel pairs in the same topologically

881 associated domain (TAD), we used publicly-available TADs from human liver ${ }^{28}$. We

882 compared the number of colocalizations with the sentinel variant and colocalized gene in the 
883 same TAD divided by all colocalizations in which the sentinel variant lies in a TAD. To test

884 if this ratio was statistically significant, we generated random TAD boundaries using

885 'bedtools shuffle' 1000 times, and calculated the same ratio for these randomly-generated

886 TAD boundaries.

887

888 Pathway enrichment

889

890 We used ClusterProfiler v3.6.0 ${ }^{29}$ to look for pathways over-represented in each gene list:

891 genes with eQTL colocalization and genes interacting with variants in GWAS credible sets.

892 We used the enrichKEGG function to look for pathway enrichment in KEGG pathways

893 (using the latest version of the KEGG database ${ }^{30}$ ). We first re-mapped gencode IDs to gene

894 symbols using the Gencode v24 annotation and then used the biomaRt R package v2.34.2 31

895 to convert gene symbols to Entrez IDs. We ran enrichKEGG to identify enriched pathways

896 significant at a Benjamini-Hochberg threshold of 0.05.

897

898

Enrichment in known lipid-associated genes

899

900 We calculated enrichment odds ratio of genes identified in our analysis with three known sets

901 of lipid-associated genes using the Fisher's exact test (R function 'fisher.test'). First, we

902 identified a list of 33 Mendelian genes from ClinVar ${ }^{32}$ with lipidemia-associated ICD10

903 codes (E78). Second, we used the set of genes identified from a transcriptome-wide

904 association study (TWAS) on the same GWAS and GTEx v8 summary statistics using the S-

905 PrediXcan software ${ }^{33}$ default setup. Third, we used 35 genes with rare-coding variants

906 associated with lipid levels ${ }^{34}$.

907 
909

910 We used LDSC version $1.0 .1^{35}$ to estimate the enrichment of heritability using GWAS

911 summary statistics in different epigenetic and transcriptomic annotations, including gene

912 expression, chromatin marks and TF binding sites. The gene expression and chromatin mark

913 annotations and the corresponding LD scores were provided as

914 'Multitissuegeneexpr1000Gv3' and 'Multitissuechromatin1000Gv3' databases in LDSC

915 software. The TF binding site annotations were extracted from ChIP-seq data of $161 \mathrm{TFs}$

916 from ENCODE, and their LD scores were estimated from 1000 Genomes Phase 3 European

917 samples using 'ldsc.py --12'. We first converted the summary statistics for each phenotype to

918 LDSC-formatted summary statistics using 'munge_sumstats.py'. Second, we ran 'ldsc.py'

919 using the baseline_v1.2 baseline model on each annotation to estimate enrichment of

920 heritability. For primary analyses, we used multi-population GWAS summary statistics and

921 LD scores estimated from 1000 Genomes Phase 3 European samples. For secondary analyses

922 on East Asian GWAS alone, we obtained EAS-specific LD scores for the same epigenomic

923 annotations ${ }^{36}$.

924

925 GREGOR analysis

926

927 We used GREGOR ${ }^{37}$ to estimate enrichment of sentinel variants for each lipid phenotype in

928 TF binding sites for 161 TFs from ENCODE compared to a null distribution of variants

929 matched for allele frequency. We ran GREGOR with default parameters, specifying 0.8 as

930 the $\mathrm{R}^{2}$ threshold, window size of $1 \mathrm{Mb}$, and 'EUR' as the population. Annotations with FDR-

931 adjusted $\mathrm{P}$-value $<0.05$ were considered significant. 
935 We overlapped our list of colocalized genes with publicly available single-cell RNA-

936 sequencing data of 8,444 cells from liver ${ }^{38}$ and 38,408 cells from adipose (Web resources) in

937 humans. For both datasets, we downloaded normalized TPM data and existing tSNE cluster

938 annotations for each cell. For each cluster, we defined median expression for each gene

939 across all cells in that cluster. Then for each cluster, we calculated the enrichment P-value for

940 our list of colocalized genes using the 'fgsea' R package v1.4.1, which looks for

941 overrepresentation of our gene list in ranked genes for each cluster ${ }^{39}$, implemented in $\mathrm{R}$

$942 \quad 3.4 .3$

\section{Results}

946 We systematically integrated lipid GWAS results ${ }^{12}$ with multiple layers of functional

947 genomic data from diverse tissues and cell types to understand regulatory mechanisms at

948 lipid-associated loci (Figure 1). Specifically, we overlaid GWAS loci with eQTL and

949 chromatin-chromatin interactions to identify causal genes. We assessed polygenic

950 enrichments of tissue-specific histone marks to prioritize relevant tissues and examined

951 GWAS loci at transcription factor (TF) binding sites to detect lipid-relevant TFs. Finally, we

952 combined all these layers to prioritize functional variants at GWAS loci, providing a holistic

953 view of gene regulation at lipid loci in relevant tissue and cell types.

954

955 Figure 1: Schematic overview of the multi-layer functional genomic analysis. We first

956 integrate GWAS summary statistics for five lipid phenotypes with eQTL and chromatin

957 interaction data to identify potential genes mediating the GWAS association, and then 
958 incorporate epigenomic annotations to identify regulatory mechanisms at these loci. For any

959 lead variant ' $X$ ', $A, B$, and C represent nearby eGenes, and SNPs around SNP X represent

960 variants in the credible set.

961

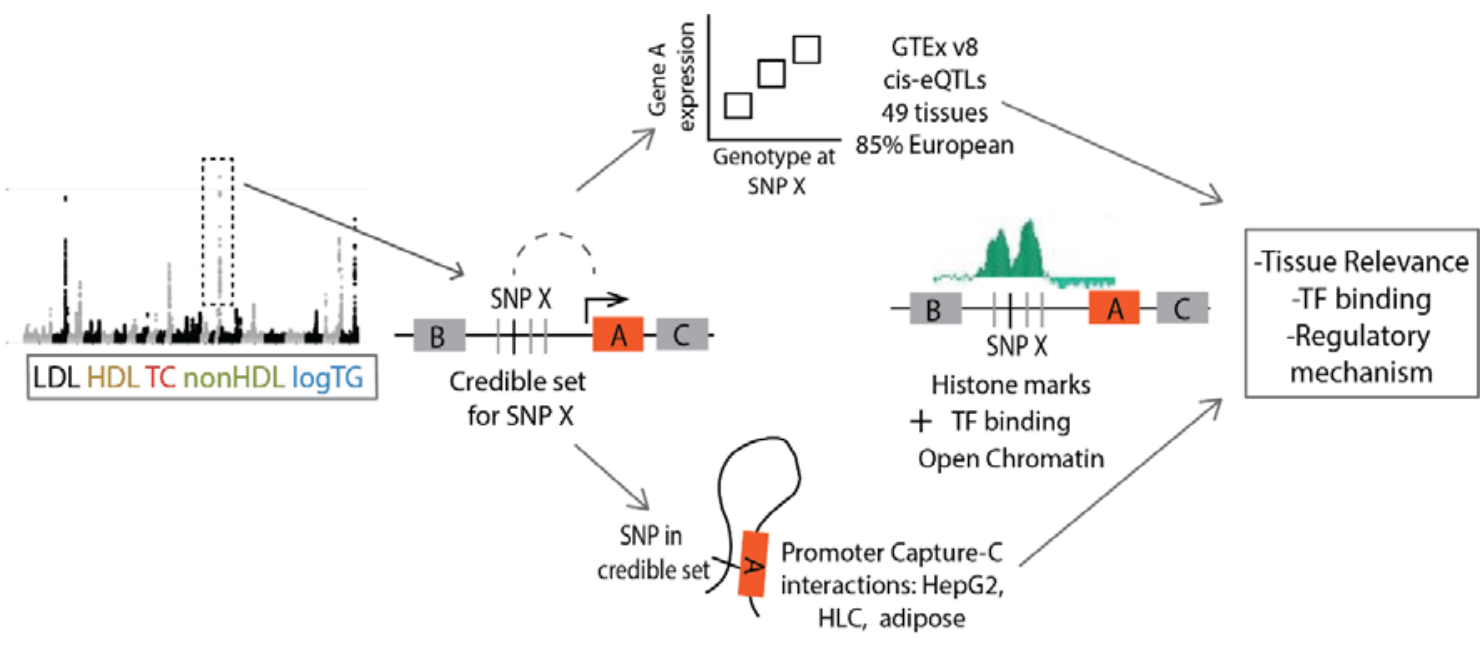

Colocalization with eQTLs identifies candidate lipid-relevant genes

965 First, we identified shared association signals between lipid levels and expression of nearby

966 genes, since most GWAS signals are presumed to influence complex traits through impact on

967 gene expression ${ }^{40}$. To do so, we tested for colocalization of each of the 1,750 significant

968 lipid GWAS association signals across the five traits examined with significant cis-eQTL

969 data across 49 human tissues from the GTEx consortium ${ }^{8}$. Here, we defined significant

970 GWAS signals as 1,750 loci reaching genome-wide significance and corrected for shadow

971 signals (Methods) in our multi-population meta-analysis for at least one of five lipid traits.

972

973 Second, we restricted our analysis to those loci likely mediated through regulatory

974 mechanisms as opposed to coding variation. In particular, we excluded all loci with credible 
975 sets containing at least one missense variant (369 of 1,750 loci, $21 \%$ of credible sets). Of the

976 remaining 1,381 GWAS loci, 696 significantly colocalized with eQTLs (the ratio of posterior

977 probability of a shared signal to the posterior probability of two signals being $>0.9^{19}$;

978 Methods) in at least one of 49 tissues for at least one lipid phenotype. This resulted in 1,076

979 colocalized eGenes ranging from 1 to 16 genes per locus (Figure 2A; Table S1). Since with

980 eQTL data alone it is difficult to disentangle a single functional gene from multiple functional

981 (and likely coregulated) genes at a locus ${ }^{41}$ we performed all downstream analyses with all

9821,076 colocalized genes, to further prioritize functional genes at loci with multiple eGenes.

983

984 To acquire additional functional insights into the 1,076 colocalized genes, we assessed their

985 enrichments across existing biological and clinical gene sets. Colocalized genes showed

986 enrichments in (a) 20 KEGG pathways ${ }^{30}$ at FDR 5\% (Table S2), including known lipid-

987 related processes such as cholesterol metabolism, PPAR signaling, and bile secretion; (b) 33

988 Mendelian genes from ClinVar ${ }^{32}$ associated with lipid-related ICD10 codes, (11 fold

989 enrichment at $\mathrm{P}=2.08 \mathrm{e}-06$, including $A P O B, L P L$, and $A P O E$; Figure $2 \mathrm{~B})$, suggesting the

990 shared genetic basis of Mendelian and complex lipid phenotypes ${ }^{42}$; (c) 35 genes with rare-

991 variant burden for lipid phenotypes in a recent multi-ancestry analysis ${ }^{34}$ (30-fold enrichment,

$992 \mathrm{P}=1.77 \mathrm{e}-16$, including $A P O B, L P L, L I P G$ and $A N G P T L 4)$, confirming shared mechanisms

993 of rare and common variation underlying lipid traits ${ }^{42,43}$. Colocalized genes also showed

994 enrichment with genes implicated in TWAS run on the same GWAS and eQTL summary

995 statistics (20-fold enrichment, $\mathrm{P}<2.22 \mathrm{e}-308)$. These enrichment results demonstrate the

996 biological relevance of candidate functional genes prioritized by our approach.

997

998 Figure 2: Overlap between eQTL colocalized genes and capture-C prioritized genes, and

999 their enrichment in known lipid-associated genes. A. Numbers of genes identified by two 
approaches: eQTL colocalization (upper half) and promoter capture-c interactions (lower

half) B. Overlap between our list of prioritized genes (left: capture-C prioritized genes; right:
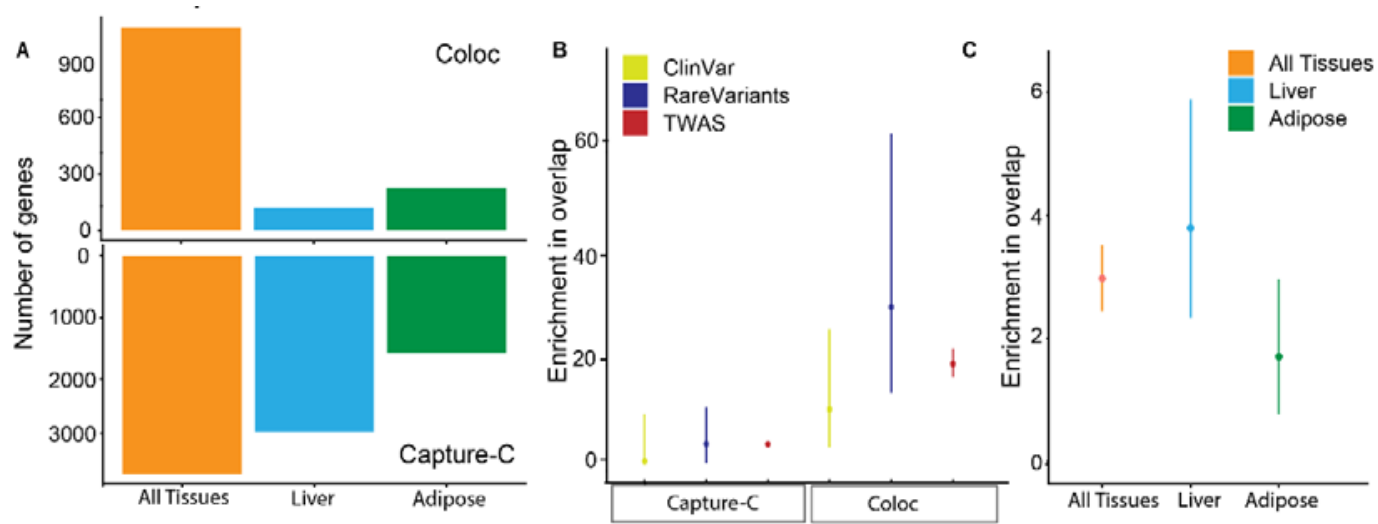

Chromatin-chromatin interactions improve eQTL-based colocalization

1012 Our eQTL-based colocalization analysis uses a linear sequence of DNA, and ignores physical 1013 interaction between non-adjacent DNA segments, another regulatory layer underlying

1014 complex human traits ${ }^{44}$. To add this layer to our analysis, we generated Capture-C data from 1015 HepG2 liver carcinoma cells (denoted as HepG2.1) and hepatocyte-like cells (HLC) derived 1016 from differentiating iPSCs (the latter is described in ${ }^{22}$ ), as well as publicly-available Capture-

1017 C datasets from HepG2 ${ }^{21,43}$ (denoted as HepG2.2) and adipose tissue ${ }^{23}$. We defined a

1018 GWAS-relevant interaction as any Capture-C interaction between any gene and a variant in 1019 the $95 \%$ credible set for a GWAS locus ${ }^{45}$. Credible set sizes ranged from 1 to 417 variants at 
1020 the 1,750 examined loci, with a median size of 5 variants per credible set. In total, 1,079

1021 GWAS loci had at least one variant in the credible set with a physical interaction with a gene 1022 promoter and 3,543 of 26,621 genes with promoter-interactions had promoters physically 1023 interacting with at least one GWAS credible set variant (Figure 2A; Table S3). Unlike eQTL1024 colocalized genes, these genes interacting with their credible sets showed limited enrichment 1025 in relevant KEGG pathways (Table S2) and lipid-related genes from ClinVar (Figure 2B), 1026 though we see 5-fold enrichment (compared to greater than 10-fold enrichment for eQTL1027 colocalized genes $)$ in genes with rare-variant lipid associations $(\mathrm{P}=2.8 \mathrm{e}-05)$ and TWAS 1028 genes $(\mathrm{P}=2.5 \mathrm{e}-288)$.

1030 Genes physically interacting with GWAS loci helped shortlist functional genes from eQTL 1031 colocalization despite their reduced enrichments in known gene sets. Of 1,079 credible sets 1032 with promoter interactions, 224 also colocalized with eQTLs for the same gene. At the gene 1033 level, 233 genes were implicated in both eQTL colocalization and Capture-C interactions 1034 (Figure 2C), representing an enrichment of 3-fold compared to random chance $(\mathrm{P}=3.11 \mathrm{e}-38)$. 1035 Among these loci with concordant eQTL colocalizations and Capture-C interactions, only $103639 \%$ of them mapped to a single gene using eQTL data alone, whereas adding Capture-C 1037 information increased this fraction to $80 \%$. These results showcase the potential value of 1038 combining eQTLs with physical chromatin interactions to prioritize functional genes at 1039 GWAS loci.

1041 Since eQTLs are likely to reside in the same topologically associated domain (TADs) as the 1042 genes they regulate ${ }^{46}$, we examined TAD structure from independent datasets at lipid GWAS 1043 loci with eQTL colocalizations. Of eQTL-GWAS colocalizations in which the sentinel 1044 variant resided within a liver $\mathrm{TAD}^{28}$, the colocalized gene resided in the same liver TAD 
$104584.8 \%$ of the time ( $\mathrm{P}<0.001$ with 1000 permutations; Methods). When we restricted

1046 colocalizations to those supported by Capture-C data in any cell type, $91.2 \%$ fall in the same

1047 TAD. These results add to the existing evidence for TAD boundaries being regulatory

1048 insulators in the cell ${ }^{47}$ and confirm our integration of chromatin interactions with eQTL

1049 colocalizations as an effective strategy to hone in on functional genes.

1050

Tissue-specific enrichment of GWAS signals differentiates lipid traits

1052

1053

Regulatory variants often affect complex traits in a tissue-specific manner ${ }^{6}$, as shown in our

1054

eQTL colocalization analysis. Specifically, by computing the ratio of the number of

1055

colocalizations in a tissue to eQTL sample size in that tissue, we found that the liver was

1056

universally enriched for colocalized eGenes with respect to sample size across all lipid traits

1057

whereas adipose was selectively enriched in HDL and TG only (Figure S1). Motivated by

1058

these findings, we leveraged systematic approaches and additional data to identify relevant

1059

tissues and cell types for each lipid trait.

1060

1061 We implemented stratified LD score regression (S-LDSC), a polygenic approach not

1062 restricted to genome-wide significant variants, on tissue-specific transcriptomic and

1063 epigenomic annotations across 204 datasets from more than 170 tissues and cell types, to

1064 identify relevant tissues for each lipid trait (Methods). Consistent with previous studies and

1065 our eQTL-based analysis, liver-related tissues (Table S4) showed strong enrichments across

1066 all lipid traits (S-LDSC enrichment p-values ranging from .001 in TG to .0001 in TC), for

1067 both expression (Figure 3A) and chromatin annotations (Figure 3B). This result was

1068 confirmed by analysis using two other approaches: DEPICT ${ }^{48}$ (Figure S2) and RSS-NET ${ }^{49}$

1069 (Table S5). To assess the robustness of our S-LDSC results based on multi-population 
1070 GWAS, we applied S-LDSC to population-specific GWAS in European and East Asian

1071 ancestry participants together with population-specific LD scores (Methods) and obtained

1072 similar results (Table S6).

1073

1074 Figure 3: Tissue relevance based on lipid GWAS and functional annotations. Partitioning

1075 heritability of GWAS summary statistics for five lipid traits on gene expression (A) and

1076 chromatin mark (B) annotations across tissues. Each plotted point represents a tested dataset

1077 for enrichment of heritability, with larger dots representing datasets with $P$-value $<0.05$;

1078 multiple annotation datasets are tested for the same tissue group. Each color represents a

1079 tissue group, and the y-axis represents -log10 P-value of enrichment of heritability.

A

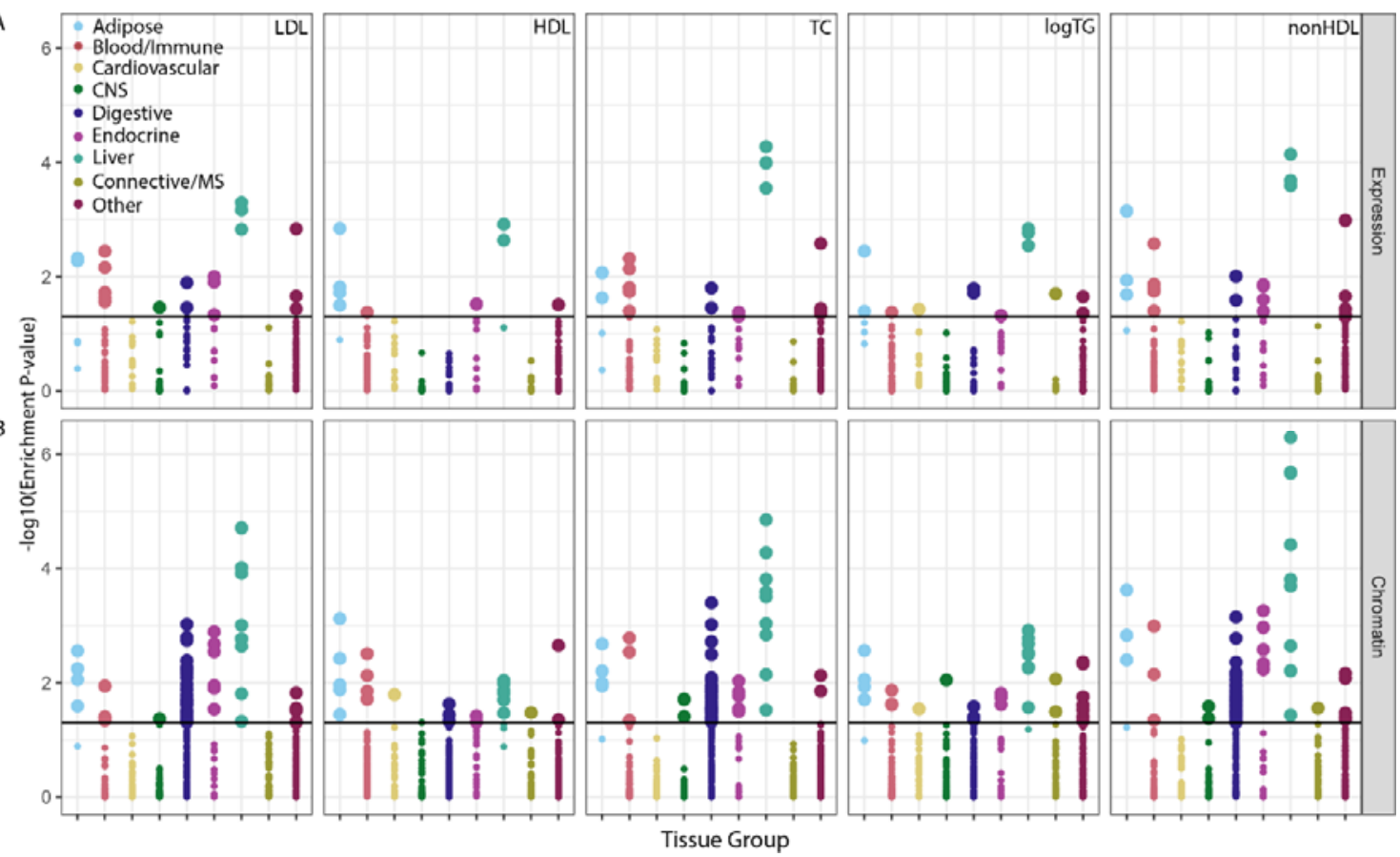

1083 The S-LDSC results also highlighted tissues selectively enriched in certain lipid traits as 1084 shown in the eQTL-based analysis. The most enriched category for HDL using chromatin annotation is 'Adipose H3K4me3' (P-value 7.6e-04); for TG, enrichment in liver-related 
1086 tissues (P-value 1.2e-03) is similar to enrichment in adipose (P-value 2.7e-03). For LDL, TC,

1087 and non-HDL, enrichment P-values for the liver were much more significant than for all

1088 other tissues including adipose (Figure 3B). We observed the same pattern in S-LDSC results

1089 based on gene expression (Figure 3A). This finding is consistent with the known influence of

1090 adipose on plasma HDL levels ${ }^{50}$, and the role of adipose as TG deposits ${ }^{51}$. These results

1091 were corroborated by eQTL colocalizations stratified by phenotype (Figure S1) and DEPICT

1092 analysis on gene expression ${ }^{48}$ (Figure S2). Together, these results confirm the liver as the

1093 tissue of action for all five lipid traits, and highlight the additional role of adipose in HDL and

1094 TG only.

1095

1096 Given the importance of the liver and adipose in modulating lipid levels, we further identified

1097 the relevant cell types within these tissues. Using existing single-cell data from adipose and

1098 liver, we performed gene-set enrichment analysis ${ }^{52}$ to identify cell-type clusters enriched for

1099 genes colocalized with any lipid trait. Out of 11 identified cell types in 20 clusters in the

1100 liver, only hepatocytes were enriched at FDR-adjusted P $<0.05$ (Figure S3), consistent with

1101 previous results ${ }^{21}$. In adipose, only adipocyte clusters and macrophage-monocyte clusters

1102 showed suggestive enrichment (nominal P < 0.05) in colocalized genes (Figure S4). Of note,

1103 the enrichment in adipocytes was significant when we restricted this analysis to genes that

1104 were colocalized only with HDL and TG (FDR-corrected P < 0.05), consistent with the

1105 selective enrichments of adipose in HDL and TG (but not the other lipid traits) from our S-

1106 LDSC analysis. Evaluations at cellular resolution are required to understand the cell-type

1107 specific mechanisms underlying lipid GWAS loci, but our results could form a useful basis

1108 for future studies.

1109

1110 Overlapping GWAS signals with binding sites highlights lipid-relevant TFs 
1112 TFs have been implicated as a key mediator of linking genetic variation to complex traits ${ }^{53}$.

1113 To understand lipid GWAS in the context of TF activity, we assessed enrichment of genome-

1114 wide significant variants at TF binding sites using GREGOR ${ }^{37}$ and performed polygenic

1115 enrichment analysis of TF binding sites using S-LDSC.

1117 Using ChIP-Seq data from 161 TFs across 91 cell types from the ENCODE project ${ }^{7}, 70.7 \%$

1118 of lipid credible sets overlapped with at least one TF binding site. Using GREGOR ${ }^{37}$, we 1119 identified 137 TFs whose binding sites were significantly enriched in GWAS lead SNPs for 1120 at least one lipid phenotype (enrichment $>2$; FDR adjusted P-value < 0.05; Figure S5; Table 1121 S7). Among these 137 enriched TFs, 69 of them (50\%) showed significant enrichments 1122 across all five lipid phenotypes, suggesting a potential core regulatory circuit shared by all 1123 lipid traits (Figure S5). The TF with the strongest enrichment in all phenotypes was ESRRA 1124 (estrogen-related receptor alpha), a nuclear receptor active in metabolic tissues ${ }^{54}$; ESRRA 1125 has been implicated in adipogenesis and lipid metabolism, and ESRRA-null mice display an 1126 increase in fat mass and obesity ${ }^{54}$.

1128 The GREGOR analysis also highlighted 68 TFs significantly enriched in specific subsets of 1129 (but not all five) lipid phenotypes (Figure S8). For example, we found 4 TFs (FOXM1, 1130 PBX3, ZKSCAN1, ZEB1) enriched in HDL and TG only, 4 TFs (EZH2, NFE2, NFATC1, 1131 KDM5A) enriched in HDL only and 11 TFs (FOSL1, IRF3, JUN, MEF2C, NANOG, 1132 PRDM1, RUNX3, SIRT6, SMC3, STAT3, ZNF217) enriched in TG only. Of these TFs, the 1133 central role of ZEB1 in adiposity ${ }^{55}$ and fat cell differentiation has been demonstrated ${ }^{56}$.

1134 Taken together, these TF-centric findings corroborate the selective enrichments of adipose in 
1135 HDL and TG (but not the other lipid traits) identified in our previous tissue prioritization

1136 analyses.

1138 Similar to tissue prioritization, we also performed polygenic enrichment analysis of TF

1139 binding sites using S-LDSC (Table S8), which differed from GREGOR analysis by looking at

1140 not only the genome-wide significant associations but also the polygenic signal irrespective

1141 of GWAS P-values. On the same 161 ENCODE TFs, this polygenic analysis identified 25

1142 TFs whose binding sites were significantly enriched in heritability (nominal $\mathrm{P}<0.05$ ) for at

1143 least one lipid phenotype (Figure S6); reassuringly, 24 of 25 TFs were also significant in

1144 GREGOR analysis. Among these enriched TFs, eight (34\%) were significantly enriched in all

1145 five lipid traits (CEBPB, CEBPD, FOXA2, HDAC2, HNF4G, NFYA, RXRA, SP1; P <

1146 0.05). Of those TFs significant in both analyses, RXRA (retinoid X receptor alpha) is also

1147 encoded by a colocalized gene (RXRA) near a GWAS hit (chr9:137,268,682). RXRA is a

1148 ligand-activated transcription factor that forms heterodimers with other receptors (including

1149 PPARG) and is involved in lipid metabolism ${ }^{57}$ and homeostasis. Moreover, 145 GWAS loci

1150 (Table S9) overlap RXRA binding peaks, suggesting that the GWAS variants might affect

1151 lipids (partially) through affecting the binding activity of RXRA. While the $R X R A$-associated

1152 variant has been previously implicated as a GWAS locus ${ }^{58}$, our study demonstrates its role in

1153 lipid biology through its regulatory influence on other lipid-associated genes.

1155 Multi-layer functional integration reveals regulatory mechanisms at GWAS loci

1157 Motivated by our finding that integrating chromatin interaction improved eQTL

1158 colocalizations, we further brought together multiple lines of functional evidence at each

1159 GWAS locus for mechanistic inference. We started with the list of genes with evidence for 
1160 both eQTL colocalization in the liver or adipose and credible set physical interactions. We

1161 next annotated each variant in the $95 \%$ credible set with various indicators of regulatory

1162 function, including its open chromatin status in liver or adipose-related cell types, its

1163 proximity to a promoter or an enhancer, and its RegulomeDB regulation probability ${ }^{59}$ (see

1164 Table S10 for the complete list of annotations used). To account for complexities of

1165 regulatory mechanisms and limitations of functional datasets, we combined evidence across

1166 these datasets to prioritize variants at GWAS loci (Figure 4A). Specifically, we prioritized

1167 variants with at least three independent lines of functional evidence (chromatin openness,

1168 physically interaction with target genes, and promoter/enhancer status in liver or adipose),

1169 with at least two being in the same tissue with colocalization with the target gene, and with a

1170 RegulomeDB score > 0.5. Applying this simple procedure to lipid GWAS we identified 13

1171 candidate loci, each with the strongest multi-layer evidence pointing to a single functional

1172 variant (Table 1). Below we describe two examples to highlight key features of this multi-

1173 layer integration framework.

1175 RRBP1 (ribosomal binding protein 1) could be identified from eQTL colocalization alone,

1176 but our multi-layer integration approach strengthened the conclusion via convergent evidence

1177 from various sources (Figure 4B). The RRBPl eQTL signals in the liver colocalize with LDL,

1178 TC, and nonHDL GWAS signals. The 'T' allele of the lead variant (chr20:17,844,684, hg19)

1179 decreases RRBPl expression levels and increases LDL, TC, and nonHDL levels. This lead

1180 variant is in open chromatin in HLC, and physically interacts with the RRBP1 promoter

1181 (250kb away) in adipose and HepG2. All these data consistently point to RRBP1 as the

1182 functional gene underlying this locus. RRBP1 specifically tethers the endoplasmic reticulum

1183 to the mitochondria in the liver--an interaction that is enriched in hepatocytes--and regulates 
1184 very low density lipoprotein (vLDL) levels ${ }^{60}$. Rare variants in $R R B P 1$ are associated with

1185 LDL in humans ${ }^{61}$ and silencing $R R B P 1$ in liver affects lipid homeostasis in mice ${ }^{60}$.

1187 Figure 4. An easy-to-implement multi-layer framework to prioritize functional variants at

1188 GWAS loci. A. Variant annotation and prioritization scheme at each credible set. B. Evidence

1189 for gene RRBP1 from functional genomics data. The LDL GWAS locus at this region is an

1190 eQTL for gene RRBP1 in the liver (second row). Variants in the credible set of this locus

1191 interact with the gene promoter in both adipose and HepG2 Capture-C data. The interacting

1192 variant is also in an open chromatin peak in three liver-related cell types. C. Multiple

1193 sources of functional genomics data support CREBRF as a gene contributing to HDL levels.

1194 The HDL GWAS locus at this region is an eQTL for gene CREBRF in adipose (second row).

1195 Variants in the credible set at this locus interact with the CREBRF promoter in adipose. The

1196 interacting variant is also in open chromatin in liver-related cell types.

A.

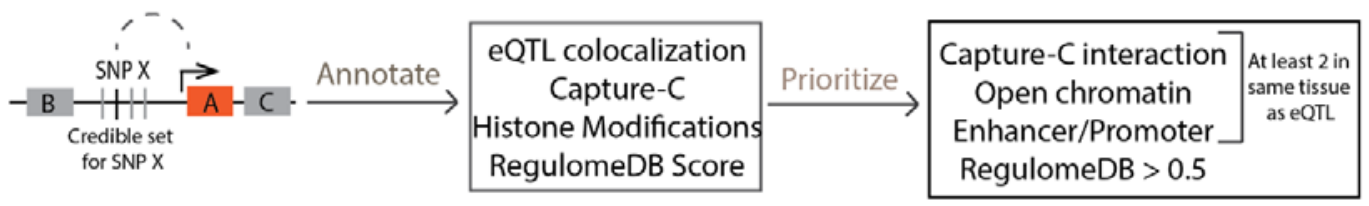

B.
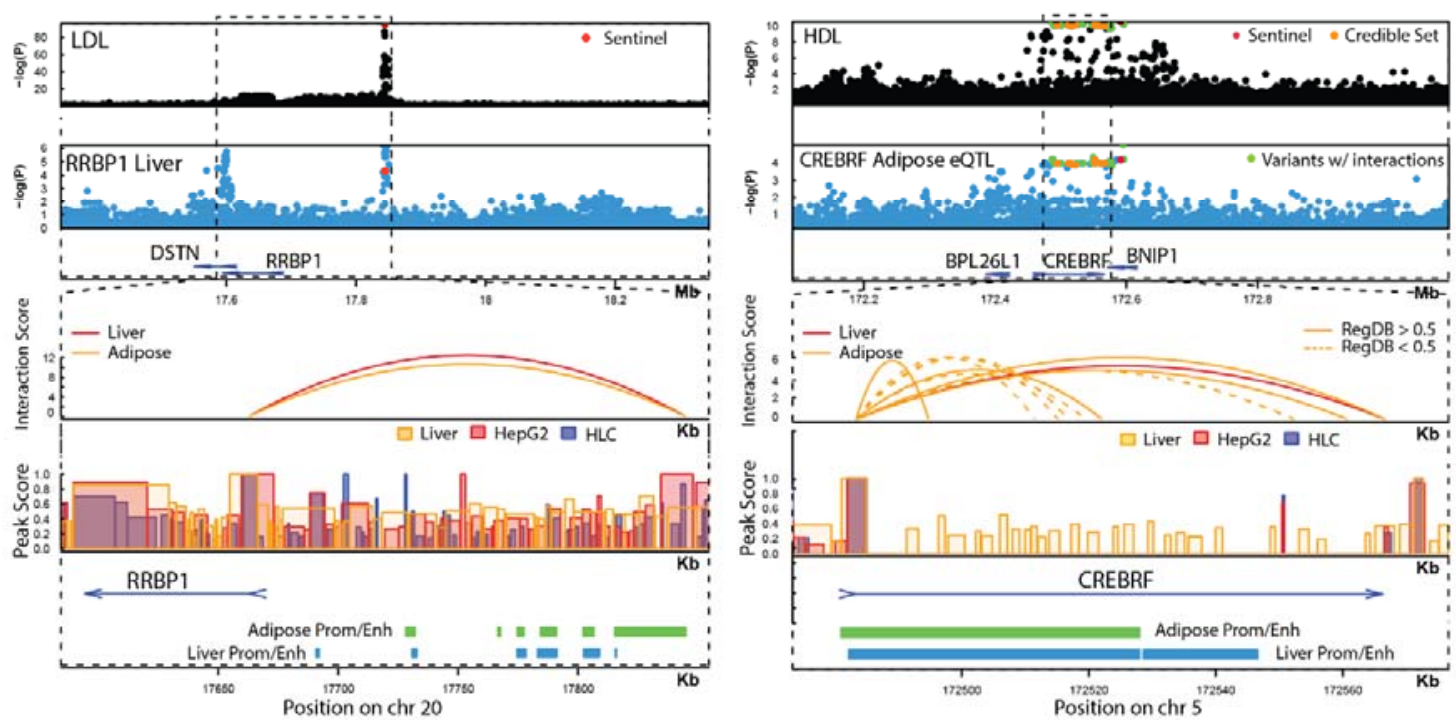

1197

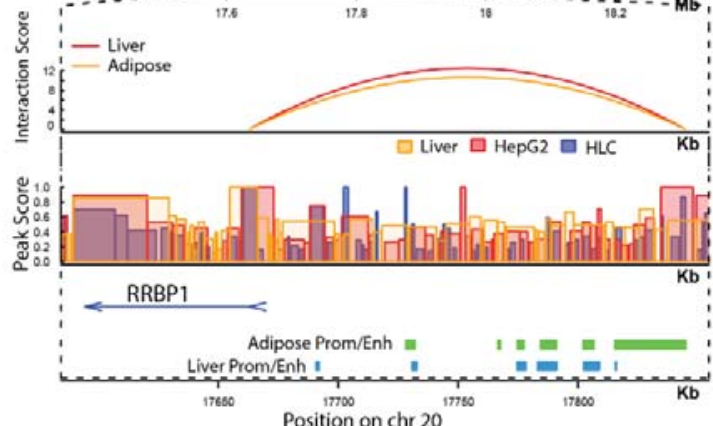

c. 
1199 CREBRF (CREB3 regulatory factor) demonstrates the power of our multi-layer integration 1200 framework in prioritizing functional variants (Figure 4C). The eQTL signals of CREBRF 1201 colocalized with a GWAS locus for HDL with 30 candidate variants. In contrast, our multi1202 layer approach identified a single candidate variant (chr5:172,566,698) at this locus that 1203 physically interacts with the $C R E B R F$ promoter in adipose, was predicted to be a regulatory 1204 element (RegulomeDB score=0.91). Consistent with the index variant (chr5:172,591,337), 1205 the allele 'A' at this functional variant increased HDL levels and increased CREBRF 1206 expression in adipose. Missense variants in $C R E B R F$ have been linked to body mass index, 1207 and the gene has been linked to obesity risk in Samoans ${ }^{62}$.

1209 Finally, to compare the power of functional fine-mapping with trans-ancestry fine-mapping, 1210 we applied our prioritization rule to credible sets derived from European-only meta-analysis.

1211 The 111 variants prioritized by our rule described above (including multiple variants in the 1212 same credible set) were all found in the multi-ancestry credible sets, representing a 3.7 fold 1213 enrichment $(\mathrm{P}<1 \mathrm{e}-04$ derived from 10000 permutations randomly sampling variants from 1214 the European-only credible sets). This convergence of complementary approaches to the 1215 same smaller set of variants highlights the power of multi-ancestry datasets as an approach to 1216 narrow in on functional variants.

\section{Discussion}

Here we integrate the largest multi-population lipid GWAS to date with a wide array of

1221 functional genomic resources to understand how noncoding genetic variation affects lipids

1222 through gene regulation. Specifically, we identify 1,076 genes whose eQTL signals 
1223 colocalize with lipid GWAS signals and demonstrate how physical chromatin interaction can

1224 improve standard eQTL-based colocalization. We assess tissue-specific enrichments of lipid

1225 GWAS signals and demonstrate the selective importance of adipose in HDL and triglyceride

1226 biology. We examine binding site enrichments of 161 TFs in lipid GWAS and expand our

1227 understanding of lipid GWAS loci (e.g., $R X R A$ ) in the context of TF activity. Finally, we

1228 build a simple and interpretable prioritization framework that automatically combines

1229 multiple lines of evidence from orthogonal datasets, pinpointing a single functional variant at

1230 each of 13 lipid-associated loci (e.g., RRBP1 and $C R E B R F$ ). While there are studies that

1231 interpret lipid GWAS associations ${ }^{21,63,64}$, the size of our multi-population GWAS and multi-

1232 layer functional integration represent a comprehensive effort and an important step forward in

1233 this direction.

1235 Our multi-layer analysis has two key strengths. First, despite a large array of functional

1236 genomic resources being embedded, our analysis produces results with high consistency. For

1237 example, the selective enrichment of adipose in HDL and TG identified by S-LDSC is

1238 confirmed by our eQTL-based colocalization and TF binding site overlap. Another example

1239 of consistency is the multi-layer prioritization of $R R B P 1$, which can be identified from eQTL-

1240 based colocalization alone and it is further validated by chromatin openness and interaction.

1241 Such convergent evidence from various sources improves the confidence of our findings.

1242 Second, our analysis highlights that combining multiple layers of regulatory information can

1243 improve sensitivity to prioritize functional genes and variants. For example, we refined eQTL

1244 colocalized genes $(1,076)$ to a smaller set of functional genes (233) through integration with

1245 promoter Capture-C data. Another example of sensitivity is CREBRF, where eQTL-based

1246 colocalization implicates 30 candidate variants and adding other regulatory layers points to a 
1247 single functional variant. Moving forward, we expect these two features will serve as useful

1248 guidelines for future integrative genomic analyses of other traits.

1250 Our results rely on the breadth and accuracy of functional genomic datasets used in our

1251 analyses. First, unlike our lipid GWAS, current functional datasets ${ }^{65}$ are limited both in

1252 sample size and ancestral diversity, which can affect discovery and replication of regulatory

1253 mechanisms in diverse populations. Second, some functional datasets are generated at limited

1254 resolution. For example, our colocalizations are based on eQTLs from bulk tissue RNA-seq ${ }^{8}$,

1255 which may miss detailed cell types and biological processes in which lipid-associated SNPs

1256 regulate gene expression ${ }^{66}$. Third, some functional datasets are not available across the full

1257 spectrum of human tissues and cell types. For example, our chromatin-chromatin interaction

1258 analysis only examines a few cell types in two known lipid-related tissues, producing results

1259 that may be biased towards known lipid biology. As more comprehensive and accurate

1260 functional genomic resources are becoming publicly available in diverse cellular contexts and

1261 ancestry groups, the resolution and power of integrative analyses like ours will be markedly

1262 increased.

1264 Other limitations of this study stem from computational methods embedded in our

1265 framework. First, the colocalization approach 'coloc' assumes one causal variant per locus,

1266 whereas recent studies suggest extensive allelic heterogeneity ${ }^{67}$ consistent with a model of a

1267 milieu of related transcription factors binding within a single locus. Accounting for allelic

1268 heterogeneity in summary statistics-based colocalization typically requires modelling

1269 multiple correlated SNPs through LD matrix ${ }^{68}$, which is computationally intensive in large-

1270 scale analyses derived from many cohorts with diverse ancestries, like the multi-population

1271 GWAS examined here. Second, due to restricted access to individual genotypes of 201 
1272 cohorts, we cannot produce multi-population LD scores within GLGC but have to use

1273 European-based LD scores in all S-LDSC analyses. This approach, though less rigorous in

1274 principle, provides robust results in practice (as confirmed by our ancestry-specific analysis),

1275 largely because $79 \%$ of cohorts in GLGC are of European descent ${ }^{12}$. That said, we caution

1276 that the same approach might fall short in ancestrally diverse studies with few European

1277 individuals ${ }^{69}$. Third, our multi-layer variant prioritization framework is built on a series of

1278 simple rules that are easy to implement on large datasets. This approach could possibly be

1279 formalized as statistical models (e.g., priors in Bayesian methods ${ }^{49}$ ), but certainly simplify

1280 computation and improve scalability of our framework. Despite the technical limitations, our

1281 approach here can serve as a useful benchmark for future development of methods with

1282 improved statistical rigor and computation efficiency.

1283 In summary, mapping noncoding genetic variation of complex traits to biological functions

1284 can benefit greatly from thorough integration of multiple layers of functional genomics, as

1285 demonstrated in the present study. Although tested on lipids only, our integrative framework

1286 is straightforward to implement more broadly on many other phenotypes, yielding functional

1287 insights of heritable traits and diseases in humans.

1288 Description of Supplemental Data

1289 Supplemental data include seven figures and ten tables, and study-specific

1290 acknowledgements.

1291 Declaration of Interests

1292 G.C-P. is currently an employee of 23andMe Inc. M.J.C. is the Chief Scientist for Genomics

1293 England, a UK Government company. B.M.P. serves on the steering committee of the Yale

1294 Open Data Access Project funded by Johnson \& Johnson. G.T., A.H., D.F.G., H.H., U.T., and 
1295 K.S. are employees of deCODE/Amgen Inc. V.S. has received honoraria for consultations

1296 from Novo Nordisk and Sanofi and has an ongoing research collaboration with Bayer Ltd.

1297 M.M. has served on advisory panels for Pfizer, NovoNordisk and Zoe Global, has received

1298 honoraria from Merck, Pfizer, Novo Nordisk and Eli Lilly, and research funding from

1299 Abbvie, Astra Zeneca, Boehringer Ingelheim, Eli Lilly, Janssen, Merck, NovoNordisk,

1300 Pfizer, Roche, Sanofi Aventis, Servier, and Takeda. M.M. and A.M. are employees of

1301 Genentech and a holders of Roche stock. M.S. receives funding from Pfizer Inc. for a project

1302 unrelated to this work. M.E.K. is employed by SYNLAB MVZ Mannheim GmbH. W.M. has

1303 received grants from Siemens Healthineers, grants and personal fees from Aegerion

1304 Pharmaceuticals, grants and personal fees from AMGEN, grants from Astrazeneca, grants

1305 and personal fees from Sanofi, grants and personal fees from Alexion Pharmaceuticals, grants

1306 and personal fees from BASF, grants and personal fees from Abbott Diagnostics, grants and

1307 personal fees from Numares AG, grants and personal fees from Berlin-Chemie, grants and

1308 personal fees from Akzea Therapeutics, grants from Bayer Vital GmbH, grants from

1309 bestbion dx GmbH, grants from Boehringer Ingelheim Pharma GmbH Co KG, grants from

1310 Immundiagnostik GmbH, grants from Merck Chemicals GmbH, grants from MSD Sharp and

1311 Dohme GmbH, grants from Novartis Pharma GmbH, grants from Olink Proteomics, other

1312 from Synlab Holding Deutschland GmbH, all outside the submitted work. A.V.K. has served

1313 as a consultant to Sanofi, Medicines Company, Maze Pharmaceuticals, Navitor

1314 Pharmaceuticals, Verve Therapeutics, Amgen, and Color Genomics; received speaking fees

1315 from Illumina, the Novartis Institute for Biomedical Research; received sponsored research

1316 agreements from the Novartis Institute for Biomedical Research and IBM Research, and

1317 reports a patent related to a genetic risk predictor (20190017119). S.K. is an employee of

1318 Verve Therapeutics, and holds equity in Verve Therapeutics, Maze Therapeutics, Catabasis,

1319 and San Therapeutics. He is a member of the scientific advisory boards for Regeneron 
1320 Genetics Center and Corvidia Therapeutics; he has served as a consultant for Acceleron, Eli

1321 Lilly, Novartis, Merck, Novo Nordisk, Novo Ventures, Ionis, Alnylam, Aegerion, Haug

1322 Partners, Noble Insights, Leerink Partners, Bayer Healthcare, Illumina, Color Genomics,

1323 MedGenome, Quest, and Medscape; he reports patents related to a method of identifying and

1324 treating a person having a predisposition to or afflicted with cardiometabolic disease

1325 (20180010185) and a genetics risk predictor (20190017119). D.K. accepts consulting fees

1326 from Regeneron Pharmaceuticals. D.O.M-K. is a part-time clinical research consultant for

1327 Metabolon, Inc. D.S. has received support from the British Heart Foundation, Pfizer,

1328 Regeneron, Genentech, and Eli Lilly pharmaceuticals. The spouse of C.J.W. is employed by

1329 Regeneron.

1331 Acknowledgments

1332 GMP, PN and CW are supported by NHLBI R01HL127564. GMP and PN are supported by 1333 R01HL142711. AG acknowledge support from the Wellcome Trust (201543/B/16/Z), 1334 European Union Seventh Framework Programme FP7/2007-2013 under grant agreement no. 1335 HEALTH-F2-2013-601456 (CVGenes@Target) \& the TriPartite Immunometabolism 1336 Consortium [TrIC]-Novo Nordisk Foundation's Grant number NNF15CC0018486. JMM is 1337 supported by American Diabetes Association Innovative and Clinical Translational Award 11338 19-ICTS-068. SR was supported by the Academy of Finland Center of Excellence in 1339 Complex Disease Genetics (Grant No 312062), the Finnish Foundation for Cardiovascular 1340 Research, the Sigrid Juselius Foundation and University of Helsinki HiLIFE Fellow and 1341 Grand Challenge grants. EW was supported by the Finnish innovation fund Sitra (EW) and 1342 Finska Läkaresällskapet. CNS was supported by American Heart Association Postdoctoral 1343 Fellowships 15POST24470131 and 17POST33650016. Charles N Rotimi is supported by 
1344 Z01HG200362. Zhe Wang, Michael Presuss, and Ruth JF Loos are supported by

1345 R01HL142302. NJT is a Wellcome Trust Investigator (202802/Z/16/Z), is the PI of the Avon

1346 Longitudinal Study of Parents and Children (MRC \& WT 217065/Z/19/Z), is supported by

1347 the University of Bristol NIHR Biomedical Research Centre (BRC-1215-2001), the MRC

1348 Integrative Epidemiology Unit (MC_UU_00011) and works within the CRUK Integrative

1349 Cancer Epidemiology Programme (C18281/A19169). Ruth E Mitchell is a member of the

1350 MRC Integrative Epidemiology Unit at the University of Bristol funded by the MRC

1351 (MC_UU_00011/1). Simon Haworth is supported by the UK National Institute for Health

1352 Research Academic Clinical Fellowship. Paul S. de Vries was supported by American Heart

1353 Association grant number 18CDA34110116. Julia Ramierz acknowledges support by the

1354 People Programme of the European Union's Seventh Framework Programme grant $\mathrm{n}^{\circ}$

1355608765 and Marie Sklodowska-Curie grant $n^{\circ}$ 786833. Maria Sabater-Lleal is supported by a

1356 Miguel Servet contract from the ISCIII Spanish Health Institute (CP17/00142) and co-

1357 financed by the European Social Fund. Jian Yang is funded by the Westlake Education

1358 Foundation. Olga Giannakopoulou has received funding from the British Heart Foundation

1359 (BHF) (FS/14/66/3129). Dr. Sander W. van der Laan is funded through grants from the

1360 Netherlands CardioVascular Research Initiative of the Netherlands Heart Foundation (CVON

1361 2011/B019 and CVON 2017-20: Generating the best evidence-based pharmaceutical targets

1362 for atherosclerosis [GENIUS I\&II]). We are thankful for the support of the FP7 EU project

1363 CVgenes@target (HEALTH-F2-2013-601456), ERA-CVD program 'druggable-MI-targets'

1364 (grant number: 01KL1802) and the Leducq Fondation 'PlaqOmics'. Study-specific

1365 acknowledgements are available in the Supplementary Material. 


\section{Web Resources}

1368 GLGC 2021 summary statistics: http://csg.sph.umich.edu/willer/public/glgc-lipids2021/

1369 GTEx v8 summary statistics: https://www.gtexportal.org/home/datasets

1370 coloc: https://cran.r-project.org/web/packages/coloc

1371 liftOver: https://genome.ucsc.edu/cgi-bin/hgLiftOver

1372 HiCUP: https://www.bioinformatics.babraham.ac.uk/projects/hicup/

1373 CHiCAGO: https://www.bioconductor.org/packages/release/bioc/html/Chicago.html

1374 GenomicRanges: https://bioconductor.org/packages/release/bioc/html/GenomicRanges.html

1375 bedtools: https://bedtools.readthedocs.io/en/latest/

1376 ClusterProfiler: https://guangchuangyu.github.io/clusterProfiler

1377 biomaRt: https://bioconductor.org/packages/release/bioc/html/biomaRt.html

1378 ClinVar: https://www.ncbi.nlm.nih.gov/clinvar/

1379 S-PrediXcan: https://github.com/hakyimlab/MetaXcan

1380 LDSC software: https://github.com/bulik/ldsc

1381 LD scores and related annotations: https://data.broadinstitute.org/alkesgroup/LDSCORE/

1382 DEPICT: https://data.broadinstitute.org/mpg/depict

1383 RSS-NET: https://github.com/SUwonglab/rss-net 
1384 Adipose single cell data:

1385 https://singlecell.broadinstitute.org/single_cell/study/SCP133/human-adipose-svf-single-cell

1386 fgsea: http://bioconductor.org/packages/release/bioc/html/fgsea.html

1387 GREGOR: https://genome.sph.umich.edu/wiki/GREGOR

1388 RegulomeDB: https://regulomedb.org/regulome-search/

1389

1390 Data and Code Availability

1391 HLC Capture-C data is available at

1392 https://www.ncbi.nlm.nih.gov/geo/query/acc.cgi?acc=GSE189026

\section{References}

1. Gallagher, M.D., and Chen-Plotkin, A.S. (2018). The Post-GWAS Era: From Association

1396 2. Cano-Gamez, E., and Trynka, G. (2020). From GWAS to Function: Using Functional

1397 Genomics to Identify the Mechanisms Underlying Complex Diseases. Front. Genet. 11, 424.

1398 3. Schaid, D.J., Chen, W., and Larson, N.B. (2018). From genome-wide associations to 1399 candidate causal variants by statistical fine-mapping. Nat. Rev. Genet. 19, 491-504.

1400 4. Smemo, S., Tena, J.J., Kim, K.-H., Gamazon, E.R., Sakabe, N.J., Gómez-Marín, C., 1401 Aneas, I., Credidio, F.L., Sobreira, D.R., Wasserman, N.F., et al. (2014). Obesity-associated 1402 variants within FTO form long-range functional connections with IRX3. Nature 507, 3711403375.

1404 5. Musunuru, K., Strong, A., Frank-Kamenetsky, M., Lee, N.E., Ahfeldt, T., Sachs, K.V., Li, 1405 X., Li, H., Kuperwasser, N., Ruda, V.M., et al. (2010). From noncoding variant to phenotype 1406 via SORT1 at the 1p13 cholesterol locus. Nature 466, 714-719.

1407 6. Hekselman, I., and Yeger-Lotem, E. (2020). Mechanisms of tissue and cell-type specificity 1408 in heritable traits and diseases. Nat. Rev. Genet. 21, 137-150.

1409 7. ENCODE Project Consortium (2012). An integrated encyclopedia of DNA elements in the 1410 human genome. Nature 489, 57-74.

1411 8. The GTEx Consortium (2020). The GTEx Consortium atlas of genetic regulatory effects 
1412 across human tissues. Science $369,1318-1330$.

1413 9. Loos, R.J.F., and Yeo, G.S.H. (2021). The genetics of obesity: from discovery to biology. 1414 Nat. Rev. Genet. doi: 10.1038/s41576-021-00414-z

1415 10. Huo, Y., Li, S., Liu, J., Li, X., and Luo, X.-J. (2019). Functional genomics reveal gene 1416 regulatory mechanisms underlying schizophrenia risk. Nat. Commun. 10, 1-19.

1417 11. Willer, C.J., Sanna, S., Jackson, A.U., Scuteri, A., Bonnycastle, L.L., Clarke, R., Heath, 1418 S.C., Timpson, N.J., Najjar, S.S., Stringham, H.M., et al. (2008). Newly identified loci that 1419 influence lipid concentrations and risk of coronary artery disease. Nat. Genet. 40, 161-169.

1420 12. Sarah E Graham, Shoa L Clarke, Kuan-Han H Wu, Stavroula Kanoni, Greg JM Zajac, 1421 Shweta Ramdas, Ida Surakka, Ioanna Ntalla, Sailaja Vedantam,, Thomas W Winkler, et al. 1422 (in press). The power of genetic diversity in genome-wide association studies of lipids. 1423 Nature. doi: 10.1038/s41586-021-04064-3

1424 13. McCarthy, S., Das, S., Kretzschmar, W., Delaneau, O., Wood, A.R., Teumer, A., Kang, 1425 H.M., Fuchsberger, C., Danecek, P., Sharp, K., et al. (2016). A reference panel of 64,976 1426 haplotypes for genotype imputation. Nat. Genet. 48, 1279-1283.

1427 14. 1000 Genomes Project Consortium, and Adam Auton, Lisa D Brooks, Richard M Durbin, 1428 Erik P Garrison, Hyun Min Kang, Jan O Korbel, Jonathan L Marchini, Shane McCarthy, Gil 1429 A McVean, Gonçalo R Abecasis (2015). A global reference for human genetic variation. 1430 Nature 526, 68-74.

1431 15. Mägi, R., Horikoshi, M., Sofer, T., Mahajan, A., Kitajima, H., Franceschini, N., 1432 McCarthy, M.I., COGENT-Kidney Consortium, T2D-GENES Consortium, and Morris, A.P. 1433 (2017). Trans-ethnic meta-regression of genome-wide association studies accounting for 1434 ancestry increases power for discovery and improves fine-mapping resolution. Hum. Mol. 1435 Genet. 26, 3639-3650.

1436 16. The International HapMap 3 Consortium (2010). Integrating common and rare genetic 1437 variation in diverse human populations. Nature 467, 52-58.

1438 17. Giambartolomei, C., Vukcevic, D., Schadt, E.E., Franke, L., Hingorani, A.D., Wallace, 1439 C., and Plagnol, V. (2014). Bayesian test for colocalisation between pairs of genetic 1440 association studies using summary statistics. PLoS Genet. 10, e1004383.

1441 18. Kuhn, R.M., Haussler, D., and Kent, W.J. (2013). The UCSC genome browser and 1442 associated tools. Brief. Bioinform. 14, 144-161.

1443 19. Çalışkan, M., Manduchi, E., Rao, H.S., Segert, J.A., Beltrame, M.H., Trizzino, M., Park, 1444 Y., Baker, S.W., Chesi, A., Johnson, M.E., et al. (2019). Genetic and Epigenetic Fine 1445 Mapping of Complex Trait Associated Loci in the Human Liver. Am. J. Hum. Genet. 105, 1446 89-107.

1447 20. Chesi, A., Wagley, Y., Johnson, M.E., Manduchi, E., Su, C., Lu, S., Leonard, M.E., 1448 Hodge, K.M., Pippin, J.A., Hankenson, K.D., et al. (2019). Genome-scale Capture C 1449 promoter interactions implicate effector genes at GWAS loci for bone mineral density. Nat. 1450 Commun. 10, 1260. 
1451 21. Selvarajan, I., Toropainen, A., Garske, K.M., López Rodríguez, M., Ko, A., Miao, Z., 1452 Kaminska, D., Õunap, K., Örd, T., Ravindran, A., et al. (2021). Integrative analysis of liver1453 specific non-coding regulatory SNPs associated with the risk of coronary artery disease. Am. 1454 J. Hum. Genet. 108, 411-430.

1455 22. Pashos, E.E., Park, Y., Wang, X., Raghavan, A., Yang, W., Abbey, D., Peters, D.T., 1456 Arbelaez, J., Hernandez, M., Kuperwasser, N., et al. (2017). Large, Diverse Population 1457 Cohorts of hiPSCs and Derived Hepatocyte-like Cells Reveal Functional Genetic Variation at 1458 Blood Lipid-Associated Loci. Cell Stem Cell 20, 558-570.e10.

23. Pan, D.Z., Garske, K.M., Alvarez, M., Bhagat, Y.V., Boocock, J., Nikkola, E., Miao, Z., Raulerson, C.K., Cantor, R.M., Civelek, M., et al. (2018). Integration of human adipocyte chromosomal interactions with adipose gene expression prioritizes obesity-related genes from 1462 GWAS. Nat. Commun. 9, 1512. Andrews, S. (2015). HiCUP: pipeline for mapping and processing Hi-C data. F1000Res. 4 , 1310.

25. Cairns, J., Freire-Pritchett, P., Wingett, S.W., Várnai, C., Dimond, A., Plagnol, V., detection of DNA looping interactions in Capture Hi-C data. Genome Biol. 17, 127.

26. Harrow, J., Frankish, A., Gonzalez, J.M., Tapanari, E., Diekhans, M., Kokocinski, F., Aken, B.L., Barrell, D., Zadissa, A., Searle, S., et al. (2012). GENCODE: the reference human genome annotation for The ENCODE Project. Genome Res. 22, 1760-1774. M.T., and Carey, V.J. (2013). Software for computing and annotating genomic ranges. PLoS Comput. Biol. 9, e1003118.

28. Leung, D., Jung, I., Rajagopal, N., Schmitt, A., Selvaraj, S., Lee, A.Y., Yen, C.-A., Lin, S., Lin, Y., Qiu, Y., et al. (2015). Integrative analysis of haplotype-resolved epigenomes across human tissues. Nature 518, 350-354. Biology 16, 284-287. the integration of genomic datasets with the R/Bioconductor package biomaRt. Nat. Protoc. 4, 1184-1191. Acids Res. 48, D835-D844.

1489 33. Barbeira, A.N., Dickinson, S.P., Bonazzola, R., Zheng, J., Wheeler, H.E., Torres, J.M., 1490 Torstenson, E.S., Shah, K.P., Garcia, T., Edwards, T.L., et al. (2018). Exploring the 
1491 phenotypic consequences of tissue specific gene expression variation inferred from GWAS

1492 summary statistics. Nat. Commun. 9, 1825.

1493 34. Hindy, G., Dornbos, P., Chaffin, M.D., Liu, D.J., Wang, M.X., Selvaraj, M.S., Zhang, D., 1494 Park, J., Aguilar-Salinas, C.A., Antonacci-Fulton, L., et al. (2021). Rare coding variants in 35 1495 genes associate with circulating lipid levels - a multi-ancestry analysis of 170,000 exomes. 1496 bioRxiv 10.1101/2020.12.22.423783

1497 35. Finucane, H.K., Bulik-Sullivan, B., Gusev, A., Trynka, G., Reshef, Y., Loh, P.-R., 1498 Anttila, V., Xu, H., Zang, C., Farh, K., et al. (2015). Partitioning heritability by functional 1499 annotation using genome-wide association summary statistics. Nat. Genet. 47, 1228.

1500 36. Kanai, M., Akiyama, M., Takahashi, A., Matoba, N., Momozawa, Y., Ikeda, M., Iwata, 1501 N., Ikegawa, S., Hirata, M., Matsuda, K., et al. (2018). Genetic analysis of quantitative traits 1502 in the Japanese population links cell types to complex human diseases. Nat. Genet. 50, 3901503400.

1504 37. Schmidt, E.M., Zhang, J., Zhou, W., Chen, J., Mohlke, K.L., Eugene Chen, Y., and 1505 Willer, C.J. (2015). GREGOR: evaluating global enrichment of trait-associated variants in 1506 epigenomic features using a systematic, data-driven approach. Bioinformatics 31, 260115072606.

38. MacParland, S.A., Liu, J.C., Ma, X.-Z., Innes, B.T., Bartczak, A.M., Gage, B.K., Manuel, 1510 J., Khuu, N., Echeverri, J., Linares, I., et al. (2018). Single cell RNA sequencing of human liver reveals distinct intrahepatic macrophage populations. Nat. Commun. 9, 4383.

1517 42. Blair, D.R., Lyttle, C.S., Mortensen, J.M., Bearden, C.F., Jensen, A.B., Khiabanian, H., 1518 Melamed, R., Rabadan, R., Bernstam, E.V., Brunak, S., et al. (2013). A nondegenerate code 1519 of deleterious variants in Mendelian loci contributes to complex disease risk. Cell 155(1):70152080.

43. Teslovich, T.M., Musunuru, K., Smith, A.V., Edmondson, A.C., Stylianou, I.M., Koseki,

1522 M., Pirruccello, J.P., Ripatti, S., Chasman, D.I., Willer, C.J., et al. (2010). Biological, clinical 1523 and population relevance of 95 loci for blood lipids. Nature 466, 707-713.

1525 Regulation and Pluripotency. Cell Stem Cell 14, 762-775.

45. Wakefield, J. (2009). Bayes factors for genome-wide association studies: comparison with P-values. Genet. Epidemiol. 33, 79-86. 
43.

1531 47. Matharu, N.K., and Ahanger, S.H. (2015). Chromatin Insulators and Topological

1532 Domains: Adding New Dimensions to 3D Genome Architecture. Genes 6, 790-811.

1533 48. Pers, T.H., Karjalainen, J.M., Chan, Y., Westra, H.-J., Wood, A.R., Yang, J., Lui, J.C., 1534 Vedantam, S., Gustafsson, S., Esko, T., et al. (2015). Biological interpretation of genome1535 wide association studies using predicted gene functions. Nat. Commun. 6, 5890.

1536 49. Zhu, X., Duren, Z., and Wong, W.H. (2021). Modeling regulatory network topology 1537 improves genome-wide analyses of complex human traits. Nat. Commun. 12, 2851.

1538 50. Zhang, T., Chen, J., Tang, X., Luo, Q., Xu, D., and Yu, B. (2019). Interaction between 1539 adipocytes and high-density lipoprotein:new insights into the mechanism of obesity-induced 1540 dyslipidemia and atherosclerosis. Lipids Health Dis. 18, 223.

1541 51. A. D. Sniderman, K. Cianflone, P. Arner, L. K. M. Summers, and K. N. Frayn (1998) The 1542 Adipocyte, Fatty Acid Trapping, and Atherogenesis. Arteriosclerosis, Thrombosis, and 1543 Vascular Biology. 18:147-151.

1544 52. Subramanian, A., Tamayo, P., Mootha, V.K., Mukherjee, S., Ebert, B.L., Gillette, M.A., 1545 Paulovich, A., Pomeroy, S.L., Golub, T.R., Lander, E.S., et al. (2005). Gene set enrichment 1546 analysis: a knowledge-based approach for interpreting genome-wide expression profiles. 1547 Proc. Natl. Acad. Sci. U. S. A. 102, 15545-15550.

1548 53. Degtyareva, A.O., Antontseva, E.V., and Merkulova, T.I. (2021). Regulatory SNPs: 1549 Altered Transcription Factor Binding Sites Implicated in Complex Traits and Diseases. Int. J. 1550 Mol. Sci. 22,.

1551 54. Tripathi, M., Yen, P.M., and Singh, B.K. (2020). Estrogen-Related Receptor Alpha: An 1552 Under-Appreciated Potential Target for the Treatment of Metabolic Diseases. Int. J. Mol. Sci. 155321,

1554 55. Saykally, J.N., Dogan, S., Cleary, M.P., and Sanders, M.M. (2009). The ZEB1

1555 Transcription Factor Is a Novel Repressor of Adiposity in Female Mice. PLoS One 4, e8460.

1556 56. Gubelmann, C., Schwalie, P.C., Raghav, S.K., Röder, E., Delessa, T., Kiehlmann, E., 1557 Waszak, S.M., Corsinotti, A., Udin, G., Holcombe, W., et al. (2014). Identification of the 1558 transcription factor ZEB1 as a central component of the adipogenic gene regulatory network. 1559 Elife 3, e03346.

1560 57. Neuschwander-Tetri, B.A. (2015). Retinoid X receptor: the forgotten partner in regulating 1561 lipid metabolism? Am. J. Clin. Nutr. 102, 5-6.

1562 58. Peloso, G.M., Demissie, S., Collins, D., Mirel, D.B., Gabriel, S.B., Cupples, L.A., 1563 Robins, S.J., Schaefer, E.J., and Brousseau, M.E. (2010). Common genetic variation in 1564 multiple metabolic pathways influences susceptibility to low HDL-cholesterol and coronary 1565 heart disease. J. Lipid Res. 51, 3524-3532.

1566 59. Boyle, A.P., Hong, E.L., Hariharan, M., Cheng, Y., Schaub, M.A., Kasowski, M., 1567 Karczewski, K.J., Park, J., Hitz, B.C., Weng, S., et al. (2012). Annotation of functional 1568 variation in personal genomes using RegulomeDB. Genome Research 22, 1790-1797. 
1569 60. Anastasia, I., Ilacqua, N., Raimondi, A., Lemieux, P., Ghandehari-Alavijeh, R., Faure, G., 1570 Mekhedov, S.L., Williams, K.J., Caicci, F., Valle, G., et al. (2021). Mitochondria-rough-ER 1571 contacts in the liver regulate systemic lipid homeostasis. Cell Rep. 34, 108873.

1572 61. Jurgens, S.J., Choi, S.H., Morrill, V.N., Chaffin, M., Pirruccello, J.P., Halford, J.L., 1573 Weng, L.-C., Nauffal, V., Roselli, C., Hall, A.W., et al. (2020). Rare Genetic Variation 1574 Underlying Human Diseases and Traits: Results from 200,000 Individuals in the UK 1575 Biobank.

1576 62. Minster, R.L., Hawley, N.L., Su, C.-T., Sun, G., Kershaw, E.E., Cheng, H., Buhule, O.D., 1577 Lin, J., Reupena, M.S., Viali, S. 'itea, et al. (2016). A thrifty variant in CREBRF strongly 1578 influences body mass index in Samoans. Nat. Genet. 48, 1049-1054.

1579 63. Klarin, D., Damrauer, S.M., Cho, K., Sun, Y.V., Teslovich, T.M., Honerlaw, J., Gagnon, 1580 D.R., DuVall, S.L., Li, J., Peloso, G.M., et al. (2018). Genetics of blood lipids among $1581 \sim 300,000$ multi-ethnic participants of the Million Veteran Program. Nat. Genet. 50, 151415821523.

64. Li, Z., Votava, J.A., Zajac, G.J.M., Nguyen, J.N., Leyva Jaimes, F.B., Ly, S.M., Brinkman, J.A., De Giorgi, M., Kaul, S., Green, C.L., et al. (2020). Integrating Mouse and Human Genetic Data to Move beyond GWAS and Identify Causal Genes in Cholesterol 1586 Metabolism. Cell Metab. 31, 741-754.e5. Parker, S.C.J. (2019). Cell Specificity of Human Regulatory Annotations and Their Genetic 1589 Effects on Gene Expression. Genetics 211, 549-562.

66. van der Wijst, M.G.P., de Vries, D.H., Groot, H.E., Trynka, G., Hon, C.C., Bonder, M.J., Stegle, O., Nawijn, M.C., Idaghdour, Y., van der Harst, P., et al. (2020). Science Forum: The 1592 single-cell eQTLGen consortium. eLife 2020;9:e52155.

67. Arvanitis, M., Tayeb, K., Strober, B.J., and Battle, A. (2021). Redefining tissue specificity of genetic regulation of gene expression in the presence of allelic heterogeneity. medRxiv 2021.06.28.21259545. summary statistics from genome-wide association studies. Ann. Appl. Stat. 11, 1561-1592. 


\section{Tables}

Table 1. Thirteen prioritized loci with highest confidence of a single functional variant in the credible set. The 'sentinel' column represents the lead variant at the locus. 'Prioritized var' represents the prioritized variant in the credible set. Columns 5-8 represent overlap of the functional variant with open chromatin ('Open'), capture-C ('CapC') interactions with the candidate gene, enhancer and promoter marks from Roadmap in liver ('Liver'), adipose ('Ad'), both or none of these datasets. The 'RegDB' column represents the RegulomeDB score of the prioritized variant.

\begin{tabular}{|c|c|c|c|c|c|c|c|c|}
\hline $\begin{array}{l}\text { Gene } \\
\text { Name }\end{array}$ & Tissue & Sentinel & $\begin{array}{c}\text { Prioritized } \\
\text { Var }\end{array}$ & Open & CapC & $\begin{array}{c}\text { Enhance } \\
\mathbf{r} \\
\end{array}$ & $\begin{array}{c}\text { Promote } \\
\text { r } \\
\end{array}$ & RegDB \\
\hline СЕР68 & Adipose & $2: 65284231$ & 65279414 & Liver & Liver & None & Ad & 0.5896 \\
\hline TIPARP & Adipose & $3: 156797941$ & 156795408 & Both & Both & Ad & Liver & 0.705 \\
\hline$C R E B R F$ & Adipose & $5: 172591337$ & 172566698 & Liver & Ad & None & Both & 0.9124 \\
\hline PALM2 & Adipose & $9: 112556911$ & 112556911 & Both & Ad & Both & None & 0.6091 \\
\hline$M E G F 9$ & Adipose & $9: 123481206$ & 123421556 & Liver & Ad & None & Liver & 0.9933 \\
\hline$G B F 1$ & Liver & 10:104142294 & 104107191 & Ad & Ad & Both & Ad & 0.705 \\
\hline$M I C A L 2$ & Liver & $11: 12071855$ & 12221016 & Liver & Liver & Liver & Ad & 0.6018 \\
\hline$A C P 2$ & Liver & 11:47278917 & 47276350 & Ad & Liver & Liver & Ad & 0.6091 \\
\hline PTPRJ & Adipose & $11: 48021778$ & 48011180 & Liver & Ad & Liver & Ad & 0.8797 \\
\hline $\begin{array}{c}\text { NFATC } 2 I \\
P\end{array}$ & Adipose & 16:28899411 & 28883327 & Liver & Liver & None & Both & 0.6091 \\
\hline$H E L Z$ & Liver & $17: 65109591$ & 65156919 & Liver & Liver & Both & Ad & $\begin{array}{c}0.6090 \\
6\end{array}$ \\
\hline FAM210A & Liver & $18: 13725674$ & 13725674 & Liver & Liver & Both & Ad & 0.7571 \\
\hline$R R B P 1$ & Liver & $20: 17844684$ & 17844684 & Both & Ad & Both & Ad & 0.6091 \\
\hline
\end{tabular}

